\title{
CYCLE SPACES OF FLAG DOMAINS ON GRASSMANNIANS AND RIGIDITY OF HOLOMORPHIC MAPPINGS
}

\author{
Sui-Chung NG
}

\begin{abstract}
We study certain cycle spaces on flag domains of $S U(p, q)$-type on Grassmannians and their relations with the rigidity of holomorphic mappings between Grassmannians. Rigidity is obtained for those holomorphic maps that preserve the distinguished boundaries of these flag domains. Our results cover in particular certain special cases of Baouendi-Huang's Theorem on local proper holomorphic mappings between generalized balls of equal signature.
\end{abstract}

\section{Introduction}

1.1. Background and statement of results. In this paper, we study certain cycle spaces on a particular type of flag domains on Grassmannians and their relations with the rigidity of holomorphic mappings between Grassmannians. There is an extensive literature on the studies of cycle spaces of flag domains; see [1] for a comprehensive account on the subject. The structural results on these cycle spaces have found many applications in other areas, for instance, the moduli problems on marked K3 surfaces and variations of Hodge structures. The main purpose of the current paper is to illustrate how the structure of cycle spaces may lead to rigidity of holomorphic mappings between flag domains and their ambient rational homogeneous spaces.

Denote by $G_{k+1, n-k}$ the Grassmannian of $(k+1)$-planes in $\mathbb{C}^{n+1}$. Let $\ell \in\{0, \ldots, n-1\}$, we equip $\mathbb{C}^{n+1}$ with the standard non-degenerate Hermitian form $H_{\ell+1}$ of signature $(\ell+1, n-\ell)$, where $\ell+1$ eigenvalues are 1 and the other $n-\ell$ eigenvalues are -1 . The flag domains we are looking at are the domains in $G_{k+1, n-k}$ corresponding to the $(k+1)$-planes in $\mathbb{C}^{n+1}$ on which the restriction of $H_{\ell+1}$ is positive definite. (In particular, we require that $\ell \geq k$.) They are open orbits on $G_{k+1, n-k}$ under the actions of the real forms $S U(\ell+1, n-\ell)$ of $S L(n+1, \mathbb{C})$ which act on $G_{k+1, n-k}$ as groups of biholomorphisms. We denote these domains by $\mathbb{D}_{k, n}^{\ell}$. In the later section, we will also give a definition of $\mathbb{D}_{k, n}^{\ell}$ using the homogeneous coordinates in $G_{k+1, n-k}$. When $\ell=k, \mathbb{D}_{k, n}^{\ell}=\mathbb{D}_{k, n}^{k}$ can be recognized as the type-I irreducible bounded symmetric domain $\Omega_{k+1, n-k}$ embedded in $G_{k+1, n-k}$ by the Borel embedding. We will exclude this case because the domain (being a domain in a Euclidean space) does not contain any compact subvarieties (cycles) of non-zero dimension. In addition, we actually need the cycle spaces to extend across the boundary, more precisely, we will look at those flag domains whose boundaries also contain similar compact subvarieties as the domains. For this reason, we will also exclude the cases, where $\ell \geq n-k-1$. Thus, in summary, we will be dealing with the domains $\mathbb{D}_{k, n}^{\ell} \subset G_{k+1, n-k}$, where $k+1 \leq \ell \leq n-k-2$.

Received by the editors September 28, 2011.

2000 Mathematics Subject Classification. xxx. 
Since the boundaries of the flag domains play a crucial role in the study, we will first make some general remarks on their structures. For each fixed $\mathbb{D}_{k, n}^{\ell}$, its boundary stratifies into $k+1$ orbits under the action of $S U(\ell+1, n-\ell)$. We denote these strata by $\partial_{r} \mathbb{D}_{k, n}^{\ell}, 1 \leq r \leq k+1$, where each $\partial_{r} \mathbb{D}_{k, n}^{\ell}$ is the orbit of $S U(\ell+1, n-\ell)$ corresponding to the set of $(k+1)$-planes in $\mathbb{C}^{n+1}$ on which the restriction of $H_{\ell+1}$ is semi-positive definite with the dimension of the null space being $r$. The most important stratum for us is $\partial_{k+1} \mathbb{D}_{k, n}^{\ell}$ and we will also denote it by $\Sigma\left(\mathbb{D}_{k, n}^{\ell}\right)$. Note that for $\mathbb{D}_{k, n}^{k}$, which is a bounded symmetric domain, $\Sigma\left(\mathbb{D}_{k, n}^{k}\right)$ is just the Shilov boundary. We can now state our main result.

Theorem 1.1. Let $\ell \in\{k+1, \ldots, n-k-2\}$. Let $U \subset G_{k+1, n-k}$ be a connected open set such that $U \cap \Sigma\left(\mathbb{D}_{k, n}^{\ell}\right) \neq \emptyset$. Suppose $f: U \rightarrow G_{k+1, m-k}$ is a holomorphic embedding in one of the following cases:

(i) $k+1 \leq \ell<\frac{n}{2}, f\left(U \cap \Sigma\left(\mathbb{D}_{k, n}^{\ell}\right)\right) \subset \Sigma\left(\mathbb{D}_{k, m}^{\ell}\right)$;

(ii) $\frac{n}{2} \leq \ell \leq n-k-2, f\left(U \cap \Sigma\left(\mathbb{D}_{k, n}^{\ell}\right)\right) \subset \Sigma\left(\mathbb{D}_{k, m}^{\ell+m-n}\right)$,

then $f$ extends to a standard embedding of $G_{k+1, n-k}$ into $G_{k+1, m-k}$. If furthermore, we have either

(i) $k+1 \leq \ell<\frac{n}{2}, f\left(U \cap \mathbb{D}_{k, n}^{\ell}\right) \subset \mathbb{D}_{k, m}^{\ell} ;$ or

(ii) $\frac{n}{2} \leq \ell \leq n-k-2, f\left(U \cap \mathbb{D}_{k, n}^{\ell}\right) \subset \mathbb{D}_{k, m}^{\ell+m-n}$,

then the extension restricting on $\mathbb{D}_{k, n}^{\ell}$ is a proper holomorphic embedding into $\mathbb{D}_{k, m}^{\ell}$ in case $(i)$ and $\mathbb{D}_{k, m}^{\ell+m-n}$ in case $(i i)$.

In the above theorem, by a standard embedding between two Grassmannians, we mean an embedding induced by a linear embedding of the corresponding Euclidean spaces.

For $k=0$, Theorem 1.1 covers half of the cases in the following theorem of Baouendi-Huang [2] on the rigidity of Cauchy-Riemann mappings between real hyperquadrics with positive signatures in projective spaces. To simplify the notations, we will write $\mathbb{D}_{n}^{\ell}$ for the flag domains $\mathbb{D}_{0, n}^{\ell}$ on $G_{1, n}=\mathbb{P}^{n}$ (In [2], the notation is $\left.\mathbb{B}_{\ell}^{n}\right)$.

Theorem 1.2 (Baouendi-Huang). Let $1 \leq \ell \leq n-2$ and $U \subset \mathbb{P}^{n}$ be a connected open set such that $U \cap \partial \mathbb{D}_{n}^{\ell} \neq \emptyset$. Suppose $f: U \rightarrow \mathbb{P}^{m}$ is a holomorphic mapping satisfying either $(i) f\left(U \cap \partial \mathbb{D}_{n}^{\ell}\right) \subset \partial \mathbb{D}_{m}^{\ell}$; or $(i i) f\left(U \cap \partial \mathbb{D}_{n}^{\ell}\right) \subset \partial \mathbb{D}_{m}^{\ell+m-n}$, then $f$ extends to a totally geodesic embedding of $\mathbb{P}^{n}$ into $\mathbb{P}^{m}$. If furthermore, we have either in case $(i) f\left(U \cap \mathbb{D}_{n}^{\ell}\right) \subset \mathbb{D}_{m}^{\ell}$; or in case $($ ii $) f\left(U \cap \mathbb{D}_{n}^{\ell}\right) \subset \mathbb{D}_{m}^{\ell+m-n}$, then the extension restricting on $\mathbb{D}_{n}^{\ell}$ is a proper holomorphic embedding into $\mathbb{D}_{m}^{\ell}$ in case $(i)$ and into $\mathbb{D}_{m}^{\ell+m-n}$ in case $(i i)$.

In the first half of the paper, we single out the case $k=0$, i.e., the flag domains $\mathbb{D}_{n}^{\ell}$ in the projective space $\mathbb{P}^{n}$. The reason for this is twofold. On the one hand, the cycles being considered in this case are linear subvarieties in $\mathbb{P}^{n}$ whose structures and description are easier and hence better for illustrating the basic principles employed in the 
paper. On the other hand, there are some major differences between the extension theorems on the projective spaces and on the Grassmannians of higher rank that we are going to apply. These differences will make themselves transparent later in the paper.

We now give the scheme of proof of Theorem 1.1 for $k=0$ in case (i). Case (ii) is similar. We first determine the set of maximal linear subvarieties contained in $\mathbb{D}_{n}^{\ell}$ and in its boundary. They will be shown to be of dimension $\ell$. The corresponding cycle space turns out to be the closure $\overline{\mathbb{D}}_{\ell, n}^{\ell}$ in $G_{\ell+1, n-\ell}$, which is just the closure of the typeI bounded symmetric domain $\Omega_{\ell+1, n-\ell}$ in $\mathbb{C}^{(\ell+1)(n-\ell)}$. This can be obtained by recalling how the domains $\mathbb{D}_{n}^{\ell}$ are defined using the non-degenerate Hermitian forms on $\mathbb{C}^{n+1}$. However, in the paper we use explicit matrix computations to obtain the result. This first of all provides us with an easy way to specify a particular linear subvariety and is at the same time important for our analysis on the boundary. After determining the cycle space of maximal linear subvarieties, we show that any maximal linear subvariety in the boundary must be mapped into some maximal linear subvariety in the boundary of the target domain by the holomorphic maps satisfying the stated assumptions in the theorem. Then the main idea is to apply a function theoretic lemma to the cycle space and deduce that the maximal linear subvarieties in the domain must also be mapped into the maximal linear subvarieties in the target domain. This implies that lines in the domain are mapped to lines in the target and then we can apply a standard extension theorem on holomorphic geometric structures to conclude the proof.

When $k \geq 1$, we basically follow the same principle and we need to study the structures of certain totally geodesic sub-Grassmannians in $\mathbb{D}_{k, n}^{\ell}$. By demonstrating the preservation of these sub-Grassmannians under a mapping satisfying the hypotheses, we deduce that such a mapping preserves a distinguished set of tangent directions at each point on the flag domain. Then the last step is to invoke a Cartan-Fubini type extension theorem of Mok [3] on the characterization of standard embeddings between Grassmannians.

It could be interesting to put Theorem 1.1 in another perspective. As has been mentioned the boundary stratum $\Sigma\left(\mathbb{D}_{k, n}^{\ell}\right)$ is a generalization of the Shilov boundary of bounded symmetric domains to the flag domains $\mathbb{D}_{k, n}^{\ell}$. We recall first of all the following theorem of Alexander [4] (for rank = 1) and Henkin-Tumanov [5] (for rank $\geq 2$ ) on local characterizations of the automorphisms of irreducible bounded symmetric domains of dimension at least two in relation to the Shilov boundary.

Theorem 1.3 (Alexander, Henkin-Tumanov). Let $N \geq 2, \Omega \Subset \mathbb{C}^{N}$ be an irreducible bounded symmetric domain and $S(\Omega)$ be its Shilov boundary. Let $U \subset \mathbb{C}^{N}$ be a connected open set such that $U \cap S(\Omega) \neq \emptyset$. Suppose $f: U \rightarrow \mathbb{C}^{N}$ is a local biholomorphism satisfying $f(U \cap \Omega) \subset \Omega$ and $f(U \cap S(\Omega)) \subset S(\Omega)$, then $f$ extends to an automorphism of $\Omega$.

Now the equidimensional case of Theorem 1.1 can be regarded as a result for flag domains $\mathbb{D}_{k, n}^{\ell}$ analogous to theorems of Alexander and Henkin-Tumanov.

Corollary 1.4 (of Theorem 1.1). Let $\ell \in\{k+1, \ldots, n-k-2\}$. Let $U \subset G_{k+1, n-k}$ be a connected open set such that $U \cap \Sigma\left(\mathbb{D}_{k, n}^{\ell}\right) \neq \emptyset$. Suppose $f: U \rightarrow G_{k+1, n-k}$ is a local biholomorphism satisfying $f\left(U \cap \mathbb{D}_{k, n}^{\ell}\right) \subset \mathbb{D}_{k, n}^{\ell}$ and $f\left(U \cap \Sigma\left(\mathbb{D}_{k, n}^{\ell}\right)\right) \subset \Sigma\left(\mathbb{D}_{k, n}^{\ell}\right)$, then $f$ extends to an automorphism of $\mathbb{D}_{k, n}^{\ell}$. 
Remark. The special case (for $\Omega=\Omega_{k+1, n-k}, n \geq 2$ ) of the theorem of AlexanderHenkin-Tumanov can be incorporated to the above corollary for the case $\ell=k$. However, our method is not directly applicable to bounded symmetric domains.

1.2. Notations and definitions. We use $M(p, q ; \mathbb{C})$ to denote be the set of $p$-by- $q$ complex matrices and $M(n ; \mathbb{C}):=M(n, n ; \mathbb{C})$. For a matrix $A$, we will denote its Hermitian transpose by $A^{\mathrm{H}}$. If $A$ is a Hermitian matrix, we will write $A>0$ (resp. $A \geq 0$ ) to signify that $A$ is positive definite (resp. positive semi-definite). We use the notations $A<0$ and $A \leq 0$ similarly.

\section{Grassmannians}

Denote by $G_{p, q}$ the Grassmannian of $p$-planes in $\mathbb{C}^{p+q}$. As a Riemannian symmetric space, the rank of $G_{p, q}$ is $\min \{p, q\}$. For good notation consistency in the paper, we will almost always look at $G_{k+1, n-k}, 0 \leq k<n$, i.e., set of $(k+1)$-planes in $\mathbb{C}^{n+1}$. A matrix representative in homogeneous coordinates for a point $Z \in G_{k+1, n-k}$ is a $\operatorname{matrix}[Z] \in M(k+1, n+1 ; \mathbb{C})$,

$$
[Z]=\left[\begin{array}{ccc}
z_{1,0} & \cdots & z_{1, n} \\
\vdots & \ddots & \vdots \\
z_{k+1,0} & \cdots & z_{k+1, n}
\end{array}\right],
$$

where the row vectors of $[Z]$ constitute a basis for the $(k+1)$-plane $Z$. Matrix representatives in homogeneous coordinates are only uniquely determined up to left multiplication by non-singular matrices in $M(k+1 ; \mathbb{C})$.

\section{Flag domains}

Let $Z \in G_{k+1, n-k}$ and $[Z] \in M(k+1, n+1 ; \mathbb{C})$ be a matrix representative in homogeneous coordinates for $Z$. For $j \in\{0, \ldots, n\}$, we use the notation $[Z]=\left[Z^{\prime}, Z^{\prime \prime}\right]_{j}$ to split $[Z]$ into two block matrices with $Z^{\prime} \in M(k+1, j+1 ; \mathbb{C})$ and $Z^{\prime \prime} \in M(k+1, n-j ; \mathbb{C})$. For $\ell \in\{k, \ldots, n\}$, define

$$
\mathbb{D}_{k, n}^{\ell}=\left\{\left[Z^{\prime}, Z^{\prime \prime}\right]_{\ell} \in G_{k+1, n-k}: Z^{\prime} Z^{\prime H}-Z^{\prime \prime} Z^{\prime \prime H}>0\right\} .
$$

It is easy to see that the validity of the above matrix inequality is independent of the choice of the matrix representative in homogeneous coordinates. Moreover, it is also clear that the above definition coincide with the one appearing at the beginning of the introduction. That is, $\mathbb{D}_{k, n}^{\ell}$ is the set of $(k+1)$-planes in $\mathbb{C}^{n+1}$ on which the restriction of the non-degenerate Hermitian form $H_{\ell+1}$ is positive definite.

When $k=0$, we will write $\mathbb{D}_{0, n}^{\ell}$ as $\mathbb{D}_{n}^{\ell}$. We split the homogeneous coordinates of $G_{1, n}=\mathbb{P}^{n}$ as $\left[\mathbf{z}^{\prime}, \mathbf{z}^{\prime \prime}\right]_{\ell}$, where $\mathbf{z}^{\prime}=\left(z_{0}, \ldots, z_{\ell}\right)$ and $\mathbf{z}^{\prime \prime}=\left(z_{\ell+1}, \ldots, z_{n}\right)$. Let $\left\|\mathbf{z}^{\prime}\right\|^{2}=$ $\sum_{i=0}^{\ell}\left|z_{i}\right|^{2}$ and $\left\|\mathbf{z}^{\prime \prime}\right\|^{2}=\sum_{j=\ell+1}^{n}\left|z_{j}\right|^{2}$. Then we have

$$
\mathbb{D}_{n}^{\ell}=\left\{\left[\mathbf{z}^{\prime}, \mathbf{z}^{\prime \prime}\right]_{\ell} \in \mathbb{P}^{n}:\left\|\mathbf{z}^{\prime}\right\|^{2}>\left\|\mathbf{z}^{\prime \prime}\right\|^{2}\right\} .
$$

\section{Particular cases:}

(1) $\mathbb{D}_{k, n}^{n}=G_{k+1, n-k}$.

(2) $\mathbb{D}_{k, n}^{k} \subset G_{k+1, n-k}$ is the non-compact dual of $G_{k+1, n-k}$ as a Hermitian symmetric space. That is, $\mathbb{D}_{k, n}^{k} \cong \Omega_{k+1, n-k}$, the type-I irreducible bounded symmetric domain defined by $\left\{A \in M(k+1, n-k ; \mathbb{C}): A A^{\mathrm{H}}-I<0\right\}$. 


\section{On projective spaces}

2.1. Projective linear subspaces in $\overline{\mathbb{D}}_{n}^{\ell}$. In this section, we will determine all linear subspaces contained in $\mathbb{D}_{n}^{\ell}$ and its boundary. We will first consider the maximal linear subspaces.

2.1.1. Maximal linear subspaces. We write the homogeneous coordinates in $\mathbb{P}^{n}$ as $\left[\mathbf{z}^{\prime}, \mathbf{z}^{\prime \prime}\right]_{\ell}$. Let $L^{\prime} \subset \mathbb{P}^{n}$ (resp. $\left.L^{\prime \prime} \subset \mathbb{P}^{n}\right)$ be the linear subspace defined by the equation $\mathbf{z}^{\prime}=0$ (resp. $\left.\mathbf{z}^{\prime \prime}=0\right)$. Let also $U^{\prime}=\mathbb{P}^{n}-L^{\prime}$. Consider the projection map $\pi: U^{\prime} \rightarrow L^{\prime \prime}$ given by $\left[\mathbf{z}^{\prime}, \mathbf{z}^{\prime \prime}\right]_{\ell} \mapsto\left[\mathbf{z}^{\prime}, 0\right]_{\ell}$.

Lemma 2.1. Let $X \subset U^{\prime}$ be a compact complex-analytic subvariety. Then the projection $\left.\pi\right|_{X}: X \rightarrow L^{\prime \prime}$ is a finite map.

Proof. Fix a point $[\mathbf{a}] \in \mathbb{P}^{\ell}$ and consider the preimage $\pi^{-1}\left([\mathbf{a}, \mathbf{0}]_{\ell}\right)$. Its closure in $\mathbb{P}^{n}$ is the set $\left\{\left[z \mathbf{a}, \mathbf{z}^{\prime \prime}\right]_{\ell} \in \mathbb{P}^{n}:\left[z, \mathbf{z}^{\prime \prime}\right] \in \mathbb{P}^{n-\ell}\right\} \cong \mathbb{P}^{n-\ell}$, we denote it by $\mathbb{P}_{\mathbf{a}}^{n-\ell}$.

Let $X \subset U^{\prime}$ be a compact complex-analytic subvariety, in particular, $X \cap L^{\prime}=\emptyset$. Consider the intersection $X_{\mathbf{a}}:=X \cap \mathbb{P}_{\mathbf{a}}^{n-\ell}$. If $X_{\mathbf{a}}$ is at least one-dimensional, then $X_{\mathbf{a}} \cap L^{\prime} \neq \emptyset$ as $L^{\prime} \cap \mathbb{P}_{\mathbf{a}}^{n-\ell}$ is a hyperplane in $\mathbb{P}_{\mathbf{a}}^{n-\ell}$. This contradicts $X \cap L^{\prime}=\emptyset$.

Thus, $X_{\mathbf{a}}$ is a finite set. By the construction of $\mathbb{P}_{\mathbf{a}}^{n-\ell}$, this precisely means that the preimage $\left.\pi\right|_{X} ^{-1}\left([\mathbf{a}, \mathbf{0}]_{\ell}\right)$ is a finite set in $X$. The proof is complete.

Proposition 2.2. Let $1 \leq \ell<n$. The domain $\mathbb{D}_{n}^{\ell} \subset \mathbb{P}^{n}$ contains a family of $\ell$-dimensional projective linear subspaces. They are compact complex-analytic subvarieties in $\mathbb{D}_{n}^{\ell}$ of the maximal dimension. Moreover, the set of $\ell$-dimensional projective linear subspaces contained in $\mathbb{D}_{n}^{\ell}$ is parametrized by the points in the irreducible bounded symmetric domain $\Omega_{\ell+1, n-\ell}$.

Proof. We first give a family of projective linear subspaces $\mathbb{P}^{\ell}$ in $\mathbb{D}_{n}^{\ell}$ parametrized by $\Omega_{\ell+1, n-\ell}$, as follows. Let $A \in M(\ell+1, n-\ell ; \mathbb{C})$. Consider the $\ell$-dimensional linear subspace

$$
\left\{\left[\mathbf{z}^{\prime}, \mathbf{z}^{\prime \prime}\right]_{\ell} \in \mathbb{P}^{n}: \mathbf{z}^{\prime \prime}=\mathbf{z}^{\prime} A\right\} \cong \mathbb{P}^{\ell} \subset \mathbb{P}^{n} .
$$

Since $\mathbf{z}^{\prime} \mathbf{z}^{\prime H}>\mathbf{z}^{\prime} A A^{\mathrm{H}} \mathbf{z}^{\prime H}$ for all $\mathbf{z}^{\prime}$ if and only if $I-A A^{\mathrm{H}}>0$, we see that the above $\mathbb{P}^{\ell}$ is contained in $\mathbb{D}_{n}^{\ell}$ if and only if $A \in \Omega_{\ell+1, n-\ell}$ (see Section 1.2). In addition, it is clear that a different choice of $A$ will give a different linear subspace.

Now suppose $X$ is an irreducible compact complex-analytic subvariety contained in $\mathbb{D}_{n}^{\ell}$, in particular, $X \subset U^{\prime}$. By Lemma 2.1, the projection $\pi: X \rightarrow L^{\prime \prime}$ is a finite map and hence $\operatorname{dim}(X) \leq \operatorname{dim}\left(L^{\prime \prime}\right)=\ell$. This shows that the $\ell$-dimensional linear subspaces we constructed above are of the maximal dimension among the compact complex-analytic subvarieties of $\mathbb{D}_{n}^{\ell}$. Now if $X$ is an $\ell$-dimensional linear subspace, then $\pi: X \rightarrow L^{\prime \prime}$ a finite projection between $\ell$-dimensional linear subspaces and hence is a biholomorphism. Therefore, we have proved that any $\ell$-dimensional linear subspace contained in $\mathbb{D}_{n}^{\ell}$ is of the form $\left\{\left[\mathbf{z}^{\prime}, \mathbf{z}^{\prime \prime}\right]_{\ell}: \mathbf{z}^{\prime \prime}=\mathbf{z}^{\prime} A\right\}, A \in \Omega_{\ell+1, n-\ell}$.

From the previous proposition, we see that the points in $\Omega_{\ell+1, n-\ell}$ and the maximal linear subspaces contained in $\mathbb{D}_{n}^{\ell}$ are in one-to-one correspondence. It is natural to extend this correspondence to the closure $\bar{\Omega}_{\ell+1, n-\ell}$. In fact, by replacing the strict inequalities by non-strict inequalities, the same proof gives the following: 
Proposition 2.3. Let $1 \leq \ell<n$. The closure $\overline{\mathbb{D}}_{n}^{\ell} \subset \mathbb{P}^{n}$ contains a family of $\ell$-dimensional projective linear subspaces. They are maximal compact complex-analytic subvarieties in $\overline{\mathbb{D}}_{n}^{\ell}$. Moreover, the set of $\ell$-dimensional projective linear subspaces contained in $\overline{\mathbb{D}}_{n}^{\ell}$ is parametrized by the points in $\bar{\Omega}_{\ell+1, n-\ell}$.

We can say more about the boundary $\partial \mathbb{D}_{n}^{\ell}$ and the boundary $\partial \Omega_{\ell+1, n-\ell}$ of the cycle space.

Lemma 2.4. The maximal compact complex-analytic subvarieties in $\partial \mathbb{D}_{n}^{\ell}$ are $\ell$ dimensional projective linear subspaces when $\ell<n / 2$ and $(n-\ell-1)$-dimensional projective linear subspaces when $\ell \geq n / 2$. Moreover, any germ of a complex submanifold lying inside $\partial \mathbb{D}_{n}^{\ell}$ must be contained in one of these projective linear subspaces.

Proof. It suffices to give the proof for the case $\ell<n / 2$, because the complement of $\overline{\mathbb{D}}_{n}^{\ell}$ in $\mathbb{P}^{n}$ is $\mathbb{D}_{n}^{n-\ell-1}$ by a change of coordinates. In homogeneous coordinates $\left[z_{0}, \ldots, z_{n}\right]$, $\partial \mathbb{D}_{n}^{\ell} \subset \mathbb{P}^{n}$ is defined by the equation

$$
\sum_{i=0}^{\ell}\left|z_{i}\right|^{2}=\sum_{j=\ell+1}^{n}\left|z_{j}\right|^{2} .
$$

When $\ell<n / 2$, let $A \in M(\ell+1, n-\ell ; \mathbb{C})$ and $A A^{\mathrm{H}}=I$. It is clear that the $\ell$-dimensional linear subspace $\left\{\left[\mathbf{z}^{\prime}, \mathbf{z}^{\prime \prime}\right]_{\ell} \in \mathbb{P}^{n}: \mathbf{z}^{\prime \prime}=\mathbf{z}^{\prime} A\right\}$ is contained in $\partial \mathbb{D}_{n}^{\ell}$.

Suppose we are given a germ of a holomorphic curve $g: \Delta \rightarrow \partial \mathbb{D}_{n}^{\ell}$, where $\Delta$ is the unit disk. Without loss of generality, we may assume that $g(\Delta) \subset \partial \mathbb{D}_{n}^{\ell} \cap U$, where $U=\mathbb{P}^{n}-\left\{z_{0}=0\right\} \cong \mathbb{C}^{n}$. Expressing in inhomogeneous coordinates, $g=\left(g_{1}, \ldots, g_{n}\right)$ will satisfy the equation

$$
1+\sum_{i=1}^{\ell}\left|g_{i}\right|^{2}=\sum_{j=\ell+1}^{n}\left|g_{j}\right|^{2} .
$$

This implies $\left(g_{\ell+1}, \ldots, g_{n}\right)=\left(1, g_{1}, \ldots, g_{\ell}\right) A$ for some $A \in M(\ell+1, n-\ell ; \mathbb{C})$ satisfying $A A^{\mathrm{H}}=I$. (For an elementary proof of this fact, see [6], Lemma 4.1.) We therefore see that such a germ must be contained in one of the $\ell$-dimensional linear subspaces described above. By considering instead a holomorphic map from a higher-dimensional unit ball into $\partial \mathbb{D}_{n}^{\ell}$, the same argument works and thus the same conclusion holds for any germ of a complex submanifold contained in $\partial \mathbb{D}_{n}^{\ell}$. This in particular has also proved that those linear subspaces are maximal complex-analytic subvarieties in $\partial \mathbb{D}_{n}^{\ell}$.

Proposition 2.5. In Proposition 2.3, the boundary points of $\bar{\Omega}_{\ell+1, n-\ell}$ correspond to those projective linear subspaces intersecting $\partial \mathbb{D}_{n}^{\ell}$. Furthermore, the points on the Shilov boundary of $\bar{\Omega}_{\ell+1, n-\ell}$ correspond to those projective linear subspaces whose intersections with $\partial \mathbb{D}_{n}^{\ell}$ contain one of the maximal projective linear subspaces described in Lemma 2.4.

Proof. By comparing Propositions 2.2 and 2.3, we can already deduce the first part of the proposition. For the second part, note that the Shilov boundary of $\Omega_{\ell+1, n-\ell}$ is precisely the set of matrices $A \in \bar{\Omega}_{\ell+1, n-\ell}$ such that $A A^{\mathrm{H}}-I$ attains the lowest rank. Fix one such $A$, either we have $A A^{\mathrm{H}}=I$ (when $\ell<n / 2$ ) or there exist unitary matrices $U$ and $V$ such that $U A V^{H}=\left[\begin{array}{l}I \\ 0\end{array}\right]$ (when $\left.\ell \geq n / 2\right)$. 
For $\ell<n / 2$, the proposition follows as the $\ell$-dimensional projective linear subspace $\left\{\left[\mathbf{z}^{\prime}, \mathbf{z}^{\prime} A\right]_{\ell} \in \mathbb{P}^{n}: \mathbf{z}^{\prime} \in \mathbb{P}^{\ell}\right\}$ completely lies in $\partial \mathbb{D}_{n}^{\ell}$ if and only if $\left\|\mathbf{z}^{\prime}\right\|^{2}=\left\|\mathbf{z}^{\prime} A\right\|^{2}$ for all $\mathbf{z}^{\prime} \in \mathbb{P}^{\ell}$, which is also equivalent to $A A^{\mathrm{H}}=I$.

When $\ell \geq n / 2$, let $A \in \bar{\Omega}_{\ell+1, n-\ell}$ be a point on the Shilov boundary of $\Omega_{\ell+1, n-\ell}$, the $\ell$-dimensional projective linear subspace $\left\{\left[\mathbf{z}^{\prime}, \mathbf{z}^{\prime} A\right]_{\ell} \in \mathbb{P}^{n}: \mathbf{z}^{\prime} \in \mathbb{P}^{\ell}\right\}$ cannot lie completely inside $\partial \mathbb{D}_{n}^{\ell}$ (see Lemma 2.4). On the other hand, if we write $U A V^{H}=I^{\sharp}$, where $I^{\sharp}=\left[\begin{array}{l}I \\ 0\end{array}\right]$, after the $S U(\ell+1, n-\ell)$ transformation $\left[\mathbf{z}^{\prime}, \mathbf{z}^{\prime \prime}\right]_{\ell} \mapsto\left[\mathbf{z}^{\prime} U^{H}, \mathbf{z}^{\prime \prime} V^{H}\right]_{\ell}$, the above $\ell$-dimensional linear subspace becomes $\left\{\left[\mathbf{z}^{\prime} U^{H}, \mathbf{z}^{\prime} U^{H} I^{\sharp}\right]_{\ell}: \mathbf{z}^{\prime} \in \mathbb{P}^{\ell}\right\}$. From this form we immediately see that it contains an $(n-\ell-1)$-dimensional linear subspace which lies completely inside $\partial \mathbb{D}_{n}^{\ell}$. Since both $\mathbb{D}_{n}^{\ell}$ and $\partial \mathbb{D}_{n}^{\ell}$ are $S U(\ell+1, n-\ell)$ invariant, we have proved that the intersection of the above $\ell$-dimensional linear subspace and $\partial \mathbb{D}_{n}^{\ell}$ contains an $(n-\ell-1)$-dimensional linear subspace. Conversely, suppose the intersection of the $\ell$-dimensional linear subspace $\left\{\left[\mathbf{z}^{\prime}, \mathbf{z}^{\prime} A\right]_{\ell}: \mathbf{z}^{\prime} \in \mathbb{P}^{\ell}\right\}$ and $\partial \mathbb{D}_{n}^{\ell}$ contains an $(n-\ell-1)$-dimensional linear subspace. We can then find $n-\ell$ linearly independent vectors $\left\{\mathbf{z}_{1}^{\prime}, \ldots, \mathbf{z}_{n-\ell}^{\prime}\right\}$ in $\mathbb{C}^{\ell+1}$ such that $\left\|\mathbf{z}_{i}^{\prime}\right\|^{2}=\left\|\mathbf{z}_{i}^{\prime} A\right\|^{2}$, for every $i$. This precisely means $A A^{\mathrm{H}}-I$ has a maximal null space and hence is of the lowest rank among $A \in \bar{\Omega}_{\ell+1, n-\ell}$.

2.1.2. Linear subspaces of lower dimensions. In the above, we have determined the set of maximal linear subspaces contained in $\overline{\mathbb{D}}_{n}^{\ell}$. For the study of holomorphic mappings among $\mathbb{D}_{n}^{\ell}$, we will also determine the set of lower dimensional linear subspaces contained in $\overline{\mathbb{D}}_{n}^{\ell}$. The cycle spaces turn out to be $\overline{\mathbb{D}}_{e, n}^{\ell} \subset G_{e+1, n-e}, e \geq 1$.

Proposition 2.6. Let $1 \leq e \leq \ell$. The set of e-dimensional linear subspaces contained in $\mathbb{D}_{n}^{\ell}\left(\right.$ resp. $\left.\overline{\mathbb{D}}_{n}^{\ell}\right)$ is parametrized by $\mathbb{D}_{e, n}^{\ell}\left(\right.$ resp. $\left.\overline{\mathbb{D}}_{e, n}^{\ell}\right)$.

Proof. Every e-dimensional linear subspace $L \subset \mathbb{P}^{n}$ can be expressed in the form $L=\left\{\left[\mathbf{z} B^{\prime}, \mathbf{z} B^{\prime \prime}\right]_{\ell} \in \mathbb{P}^{n}:[\mathbf{z}] \in \mathbb{P}^{e}\right\}$ for some matrices $B^{\prime}$ and $B^{\prime \prime}$. Furthermore, $L \subset \overline{\mathbb{D}}_{n}^{\ell}$ if and only if $\left\|\mathbf{z} B^{\prime}\right\|^{2} \geq\left\|\mathbf{z} B^{\prime \prime}\right\|^{2}$ for all $[\mathbf{z}]$ which in turn is equivalent to $B^{\prime} B^{\prime H}-B^{\prime \prime} B^{\prime \prime H} \geq 0$, i.e., $\left[B^{\prime}, B^{\prime \prime}\right]_{\ell} \in \overline{\mathbb{D}}_{e, n}^{\ell}$. Thus, the set of $e$-dimensional linear subspaces contained in $\overline{\mathbb{D}}_{n}^{\ell}$ is parametrized by $\overline{\mathbb{D}}_{e, n}^{\ell}$.

Replacing non-strict inequalities by strict inequalities, the other half of the proposition follows.

2.1.3. Double fibrations. We have seen that the domain $\mathbb{D}_{n}^{\ell}$ is filled up by many linear subspaces. In general, given an integer $e \in\{1, \ldots, \ell\}$, there are many $e$-dimensional linear subspaces passing through a given point and therefore these linear subspaces do not give us a foliation of $\mathbb{D}_{n}^{\ell}$. However, if we lift everything to some Grassmann bundle over $\mathbb{D}_{n}^{\ell}$ (see the definition below), there is indeed a foliation. For illustration, we will first look at $\mathbb{D}_{n}^{1}$.

Proposition 2.7. Let $\pi: \mathbb{P T D}_{n}^{1} \rightarrow \mathbb{D}_{n}^{1}$ be the projectivized tangent bundle of $\mathbb{D}_{n}^{1}$. There is an open set $V_{n} \subset \mathbb{P} T \mathbb{D}_{n}^{1}$ with $\pi\left(V_{n}\right)=\mathbb{D}_{n}^{1}$ such that $V_{n}$ has a holomorphic $\mathbb{P}^{1}$-fibration and $V_{n} \cong \Omega_{2, n-1} \times \mathbb{P}^{1}$.

Proof. Fix a point $p \in \mathbb{D}_{n}^{1}$. Since $\mathbb{P}^{1}$ is compact and $\mathbb{D}_{n}^{1}$ is a domain, we deduce that there is an open set $U_{p} \subset \mathbb{P} T_{p} \mathbb{D}_{n}^{1}$ consisting of precisely all the projectivizations of vectors tangent to some line contained in $\mathbb{D}_{n}^{1}$. Define $V_{n}=\bigcup_{p \in \mathbb{D}_{n}^{1}} U_{p}$. Since the 
tangent direction uniquely determines a given line, combining with Proposition 2.2 when $\ell=1$, the statements in the proposition are immediate.

When $\ell \geq 2$, to obtain a similar foliation picture as in Proposition 2.7, we need to consider the more general Grassmann bundles in place of the projectivized tangent bundle. Take a point $p \in \mathbb{D}_{n}^{\ell}$ and let $T_{p} \mathbb{D}_{n}^{\ell}$ be its tangent space. The set of all $e$-planes in $T_{p} \mathbb{D}_{n}^{\ell}$ is just the Grassmannian $G_{e, n-e}$, and in the present situation we will denote it by $G_{e} T_{p} \mathbb{D}_{n}^{\ell}$. We define the $e$-Grassmann bundle $G_{e} T \mathbb{D}_{n}^{\ell}:=\bigcup_{p \in \mathbb{D}_{n}^{\ell}} G_{e} T_{p} \mathbb{D}_{n}^{\ell}$. Note that $\mathbb{P} T \mathbb{D}_{n}^{\ell}=G_{1} T \mathbb{D}_{n}^{\ell}$.

The argument in Proposition 2.7 can be directly carried over to the cases $\ell \geq 2$ and $e \in\{1, \ldots, \ell\}$ and we then have

Proposition 2.8. Let $1 \leq e \leq \ell$ and $\pi_{e}: G_{e} T \mathbb{D}_{n}^{\ell} \rightarrow \mathbb{D}_{n}^{\ell}$ be the e-Grassmann bundle of $\mathbb{D}_{n}^{\ell}$. There is an open set $\bar{V}_{e, n}^{\ell} \subset G_{e} T \mathbb{D}_{n}^{\ell}$ with $\pi_{e}\left(V_{e, n}^{\ell}\right)=\mathbb{D}_{n}^{\ell}$ such that $V_{e, n}^{\ell}$ is a holomorphic $\mathbb{P}^{e}$-bundle over $\mathbb{D}_{e, n}^{\ell}$.

We will now restrict ourselves to the case $e=\ell$. On $\mathbb{P}^{n}$, there is the tautological foliation by $\mathbb{P}^{\ell}$ on $G_{\ell} T \mathbb{P}^{n}$ and $G_{\ell} T \mathbb{P}^{n} \rightarrow G_{\ell+1, n-\ell}$ is the universal family (note that $G_{\ell+1, n-\ell}$ is the space of $\ell$-planes in $\left.\mathbb{P}^{n}\right)$. The foliation in Proposition 2.8 is certainly the restriction of the tautological foliation to the open subset $V_{\ell, n}^{\ell} \subset G_{\ell} T \mathbb{P}^{n}$. We can still add one more between these two. Let $U_{n}^{\ell}=\left\{\left[\mathbf{z}^{\prime}, \mathbf{z}^{\prime \prime}\right]_{\ell} \in \mathbb{P}^{n}: \mathbf{z}^{\prime} \neq 0\right\}$. The $\ell$-plane $\left\{\left[\mathbf{z}^{\prime}, \mathbf{z}^{\prime} A\right]_{\ell} \in \mathbb{P}^{n}:\left[\mathbf{z}^{\prime}\right] \in \mathbb{P}^{\ell}\right\}$ is contained in $U_{n}^{\ell}$ for any $A \in M(\ell+1, n-\ell ; \mathbb{C})$. Moreover, we can deduce from Lemma 2.1 that they are all the $\ell$-planes in $U_{n}^{\ell}$. Hence, as in the case of $\mathbb{D}_{n}^{\ell}$, we can find an open set $W_{\ell, n} \subset G_{\ell} T U_{n}^{\ell}$ with $\pi_{\ell}\left(W_{\ell, n}\right)=U_{n}^{\ell}$ such that $W_{\ell, n} \rightarrow \mathbb{C}^{(\ell+1)(n-\ell)}$ is a holomorphic $\mathbb{P}^{\ell}$-bundle. In fact, we have $W_{\ell, n} \cong$ $\mathbb{C}^{(\ell+1)(n-\ell)} \times \mathbb{P}^{\ell}$ by our explicit parametrization. In summary, we have the following inclusion relations:

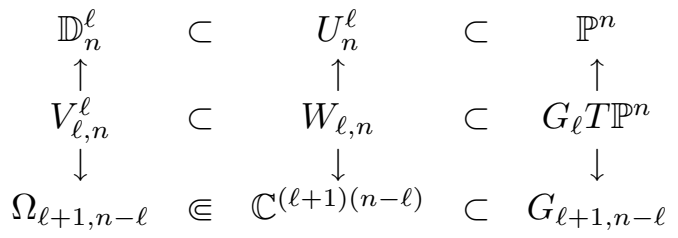

Remark. It is clear from our explicit parametrization that the inclusion relation among the cycle spaces $\Omega_{\ell+1, n-\ell} \Subset \mathbb{C}^{(\ell+1)(n-\ell)} \subset G_{\ell+1, n-\ell}$ incorporates both the classical Harish-Chandra and Borel embeddings.

2.2. Rigidity of global proper holomorphic maps. To illustrate better the basic strategy for proving rigidity, we will consider global proper holomorphic maps in this section and return to local maps in the next section. We first state two lemmas.

Lemma 2.9. Let $\Omega \Subset \mathbb{C}^{n}$ be a bounded symmetric domain and $S(\Omega) \subset \partial \Omega$ be its Shilov boundary. Let $U \subset \mathbb{C}^{n}$ be a connected open set, such that $U \cap S(\Omega) \neq \emptyset$. Suppose $u$ is a holomorphic function on $U$ and $\left.u\right|_{U \cap S(\Omega)} \equiv 0$, then $u \equiv 0$ on $U$.

Proof. We will first prove the lemma by induction for the polydisks $\Delta^{m}, m \geq 1$. For the unit disk $\Delta$, since $S(\Delta)=\partial \Delta$, the result immediately follows from the discreteness of zeros of holomorphic functions in one variable. Assume the lemma has been proved for $\Delta^{k}, 1 \leq k \leq p-1$. For $\Delta^{p} \subset \mathbb{C}^{p}$, we have $S\left(\Delta^{p}\right)=(\partial \Delta)^{p}$. Without loss of 
generality, we may assume that $U$ is a connected open neighborhood of the point $(1, \ldots, 1) \in S\left(\Delta^{p}\right)$ in $\mathbb{C}^{p}$ and we can find an open arc $\gamma \subset \partial \Delta, 1 \in \gamma$ such that $\gamma^{p} \subset U \cap S\left(\Delta^{p}\right)$. Let $\mathbf{b}_{0} \in \gamma^{p-1}$ and $L_{\mathbf{b}_{0}}=\left\{\left(z, \mathbf{b}_{0}\right) \in \mathbb{C}^{p}: z \in \mathbb{C}\right\}$. Since $u$ vanishes on $\gamma^{p}$, we have $u\left(b, \mathbf{b}_{0}\right)=0$ for $b \in \gamma$ and hence $\left.u\right|_{U \cap L_{\mathbf{b}_{0}}} \equiv 0$. In particular, $u$ vanishes on $U \cap \Delta_{\mathbf{b}_{0}}$, where $\Delta_{\mathbf{b}_{0}} \subset L_{\mathbf{b}_{0}} \cong \mathbb{C}$ is the unit disk. Now if we choose a small enough open neighborhood $B \subset \gamma^{p-1}$ of $\mathbf{b}_{0}$, then we can find an open set $A \subset \Delta$ such that $(z, \mathbf{b}) \in U \cap \Delta_{\mathbf{b}}$ for all $z \in A$ and $\mathbf{b} \in B$. Following the previous reasoning, we have $u(z, \mathbf{b})=0$ for all $z \in A$ and $\mathbf{b} \in B$. On the other hand, for a fix $z_{0} \in A$, consider $E_{z_{0}}=\left\{\left(z_{0}, \mathbf{z}\right) \in \mathbb{C}^{p}: \mathbf{z} \in \mathbb{C}^{p-1}\right\}$ and the $(p-1)$-disk $\Delta_{z_{0}}^{p-1}=\left\{\left(z_{0}, \mathbf{z}\right) \in \mathbb{C}^{p}: \mathbf{z} \in\right.$ $\left.\Delta^{p-1}\right\}$. Now since $u\left(z_{0}, \mathbf{b}\right)=0$ for $\mathbf{b} \in B \subset \gamma^{p-1} \subset S\left(\Delta^{p-1}\right)$, it follows from the induction hypothesis that $\left.u\right|_{U \cap E_{z_{0}}} \equiv 0$. By varying $z_{0} \in A$, we see that $u$ vanishes on an open set in $U$ and hence $u \equiv 0$ on $U$. We have therefore proved the lemma for the polydisks.

When $\Omega \Subset \mathbb{C}^{n}$ is a bounded symmetric domain with $\operatorname{rank}(\Omega)=r$, by the Polydisk Theorem [7], there is a totally geodesic embedding $0 \in \Delta^{r} \subset \Omega$ of the $r$-disk into $\Omega$. Moreover, if $K \subset \operatorname{Aut}(\Omega)$ is the isotropy group at $0 \in \Omega$, then $\bigcup_{k \in K} k \cdot \Delta^{r}=\Omega$. The elements in $K$ extend to unitary transformations in $\mathbb{C}^{n}$. The Shilov boundary of $\Omega$ is precisely the $K$-orbit of $S\left(\Delta^{r}\right)$, i.e., $S(\Omega)=\bigcup_{k \in K} k \cdot S\left(\Delta^{r}\right)=\bigcup_{k \in K} k$. $(\partial \Delta)^{r}$. We can choose Harish-Chandra coordinates $\left(z_{1}, \ldots, z_{n}\right)$ of $\Omega$ such that $\Delta^{r}=$ $\left\{\left(z_{1}, \ldots, z_{r}, 0, \ldots, 0\right) \in \Omega\right\}$. Without loss of generality, we may assume that $U \cap$ $S\left(\Delta^{r}\right) \neq \emptyset$. This implies in particular $U \cap \Delta^{r} \neq \emptyset$. Now take a sufficiently small open set $\mathcal{K} \subset K$ containing the identity such that $U \cap\left(k \cdot S\left(\Delta^{r}\right)\right) \neq \emptyset$ and hence also $U \cap\left(k \cdot \Delta^{r}\right) \neq \emptyset$ for all $k \in \mathcal{K}$. For each $k \in \mathcal{K}$, since $k \cdot S\left(\Delta^{r}\right) \subset S(\Omega)$, we have by assumption $\left.u\right|_{U \cap\left(k \cdot S\left(\Delta^{r}\right)\right)} \equiv 0$ and hence $\left.u\right|_{U \cap\left(k \cdot \Delta^{r}\right)} \equiv 0$ by the present lemma in the polydisk case. This implies that $u$ is identically zero when restricting on $U \cap\left(\bigcup_{k \in \mathcal{K}} k \cdot \Delta^{r}\right)$. Finally, it suffices to note that $U \cap\left(\bigcup_{k \in \mathcal{K}} k \cdot \Delta^{r}\right)$ contains an open subset of $U$ and hence $u \equiv 0$ on $U$. The proof of the lemma is complete.

Lemma 2.10. Let $U \subset \mathbb{P}^{n}$ be a connected open set. Let $h: U \rightarrow \mathbb{P}^{m}$ be a holomorphic embedding such that for every line $L$ in $\mathbb{P}^{n}$ with $L \cap U \neq \emptyset$, we have $f(U \cap L)$ being an open subset of a line in $\mathbb{P}^{m}$. Then $h$ extends to a linear embedding of $\mathbb{P}^{n}$ into $\mathbb{P}^{m}$.

Proof. See [8], for example.

Theorem 2.11. Let $1 \leq \ell<n / 2$. If $f: \mathbb{D}_{n}^{\ell} \rightarrow \mathbb{D}_{m}^{\ell}$ is a proper holomorphic map which is non-degenerate at some point, then $f$ extends to a linear embedding of $\mathbb{P}^{n}$ into $\mathbb{P}^{m}$.

Proof. The map $f: \mathbb{D}_{n}^{\ell} \rightarrow \mathbb{D}_{m}^{\ell}$ induces a meromorphic map $[d f]: G_{\ell} T \mathbb{D}_{n}^{\ell} \rightarrow G_{\ell} T \mathbb{D}_{m}^{\ell}$ by differentiation. Recalling the notations in Proposition 2.8, we restrict [ $d f]$ to $V_{\ell, n}^{\ell} \subset$ $G_{\ell} T \mathbb{D}_{n}^{\ell}$ and regard it as a map into $G_{\ell} T \mathbb{P}^{m} \supset G_{\ell} T \mathbb{D}_{m}^{\ell}$. Since $\mathbb{D}_{\ell, n}^{\ell} \cong \Omega_{\ell+1, n-\ell}$, we have $V_{\ell, n}^{\ell} \cong \Omega_{\ell+1, n-\ell} \times \mathbb{P}^{\ell}$. Thus, we are now considering the meromorphic map

$$
[d f]: \Omega_{\ell+1, n-\ell} \times \mathbb{P}^{\ell} \rightarrow G_{\ell} T \mathbb{P}^{m} .
$$

Let $\Pi: G_{\ell} T \mathbb{P}^{m} \rightarrow G_{\ell+1, m-\ell}$ be the universal family of $\ell$-planes in $\mathbb{P}^{m}$ and let $f^{\sharp}=$ $\Pi \circ[d f]$. We are going to prove that $f^{\sharp}: \Omega_{\ell+1, n-\ell} \times \mathbb{P}^{\ell} \rightarrow G_{\ell+1, m-\ell}$ is constant on each fibre $\{z\} \times \mathbb{P}^{\ell}, z \in \Omega_{\ell+1, n-\ell}$. 
At each boundary point of $\mathbb{D}_{n}^{\ell} \subset \mathbb{P}^{n}$, the Levi-form of a local defining function of $\mathbb{D}_{n}^{\ell}$ has $\ell+1 \geq 2$ negative eigenvalues, thus by Hartogs' extension, $f$ can be extended to an open neighborhood of $p$. Using the same principle (see [9], Proposition 3.2.), the global map $f$ extends to an open neighborhood $\overline{\mathbb{D}}_{n}^{\ell}$. In particular, $f^{\sharp}$ can be extended to an open neighborhood $W$ of $\bar{\Omega}_{\ell+1, n-\ell} \times \mathbb{P}^{\ell}$ in $\mathbb{C}^{(\ell+1)(n-\ell)} \times \mathbb{P}^{\ell}$ (see the paragraph after Proposition 2.8). We will still denote the extension by $f^{\sharp}$. Take a point $b$ on the Shilov boundary of $\Omega_{\ell+1, n-\ell}$. The fibre $\{b\} \times \mathbb{P}^{\ell} \subset \bar{\Omega}_{\ell+1, n-\ell} \times \mathbb{P}^{\ell}$ is the tautological lifting of an $\ell$-plane $L_{b}$ lying inside the boundary $\partial \mathbb{D}_{n}^{\ell}$ (see Proposition 2.5 and we need $1 \leq \ell<n / 2$ here). Being a proper holomorphic map, $f$ (more precisely, its extension) will map $L_{b}$ into the boundary $\partial \mathbb{D}_{m}^{\ell}$. By Lemma 2.4, $f\left(L_{b}\right)$ must be contained in an $\ell$-plane $\widetilde{L}_{b} \subset \partial \mathbb{D}_{m}^{\ell}$. From the definition of $f^{\sharp}$, it follows that $f^{\sharp}$ is constant on the fibre $\{b\} \times \mathbb{P}^{\ell}$. We have therefore proved that $f^{\sharp}$ is constant along every fibre over the Shilov boundary of $\Omega_{\ell+1, n-\ell}$.

Now pick again an arbitrary point $b$ on the Shilov boundary of $\Omega_{\ell+1, n-\ell}$ and let $U \subset \mathbb{C}^{(\ell+1)(n-\ell)}$ be a connected open set containing $b$ such that $U \times \mathbb{P}^{\ell} \subset W$. By shrinking $U$ if necessary, we can choose an open set $V \subsetneq \mathbb{P}^{\ell}$ such that $f^{\sharp}(U \times V)$ is contained in some Euclidean chart of $G_{\ell+1, m-\ell}$. Then on $U \times V$, the map $f^{\sharp}$ can be expressed in terms of holomorphic functions over some open set in a Euclidean space. We identify $U$ with a section $U \times\{v\} \subset U \times V$ and consider the derivatives of $f^{\sharp}$ in the vertical direction (i.e., along $V$ ). From the previous paragraph, we know that these vertical derivatives are zero on $U \cap S\left(\Omega_{\ell+1, n-\ell}\right)$, where $S\left(\Omega_{\ell+1, n-\ell}\right)$ is the Shilov boundary of $\Omega_{\ell+1, n-\ell}$. By Lemma 2.9 , they are identically zero on $U$. This precisely means that $f^{\sharp}$ is constant along every fibre over $U$. Now by the Identity Theorem of meromorphic functions, we can conclude that $f^{\sharp}: \Omega_{\ell+1, n-\ell} \times \mathbb{P}^{\ell} \rightarrow G_{\ell+1, m-\ell}$ is constant on each fibre $\{z\} \times \mathbb{P}^{\ell}, z \in \Omega_{\ell+1, n-\ell}$. This is equivalent to the map

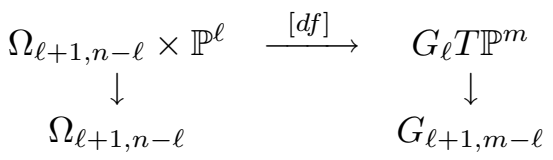

between two universal families of $\ell$-planes being fibre-preserving. Since the fibers are just the tautological liftings of the $\ell$-planes to the $\ell$-Grassmann bundles, we deduce that $f: \mathbb{D}_{n}^{\ell} \rightarrow \mathbb{D}_{m}^{\ell}$ maps each $\ell$-plane into an $\ell$-plane. Moreover, as $f$ is non-degenerate at a general point, by considering the intersections of $\ell$-planes, it follows that $f$ also maps lines into lines. Note that the lines contained in $\mathbb{D}_{n}^{\ell}$ constitute an open set in the space of lines in $\mathbb{P}^{n}$, we hence deduce further that for any line $L \subset \mathbb{P}^{n}$ intersecting $\mathbb{D}_{n}^{\ell}, f\left(\mathbb{D}_{n}^{\ell} \cap L\right)$ is an open subset of some line in $\mathbb{P}^{m}$. The theorem now follows from Lemma 2.10 .

Remark. It can be proved, like in [2], that any proper holomorphic map $f: \mathbb{D}_{n}^{\ell} \rightarrow \mathbb{D}_{m}^{\ell}$ is not totally degenerate. Therefore the non-degeneracy assumption in Theorem 2.11 can actually be removed.

Theorem 2.12. Let $\frac{n}{2} \leq \ell<n-1$ and $k \in \mathbb{N}$. If $f: \mathbb{D}_{n}^{\ell} \rightarrow \mathbb{D}_{n+k}^{\ell+k}$ is a proper holomorphic map which is non-degenerate at some point, then $f$ extends to a linear embedding of $\mathbb{P}^{n}$ into $\mathbb{P}^{n+k}$. 
Proof. The proof is similar to that of Theorem 2.11 except that we now look at the holomorphic $\mathbb{P}^{n-\ell-1}$-fibration of $G_{n-\ell-1} T \mathbb{D}_{n}^{\ell}$. By differentiation, $f$ induces a meromorphic map $[d f]: G_{n-\ell-1} T \mathbb{D}_{n}^{\ell} \rightarrow G_{n-\ell-1} T \mathbb{D}_{n+k}^{\ell+k}$. Now we restrict $[d f]$ to $V_{n-\ell-1, n}^{\ell} \subset G_{n-\ell-1} T \mathbb{D}_{n}^{\ell}$, where $V_{n-\ell-1, n}^{\ell} \rightarrow \mathbb{D}_{n-\ell-1, n}^{\ell}$ is a holomorphic $\mathbb{P}^{n-\ell-1}$ bundle. This time the cycle space $\mathbb{D}_{n-\ell-1, n}^{\ell} \subset G_{n-\ell, \ell+1}$ is no longer a bounded symmetric domain. Instead, we will see that when expressing in homogeneous coordinates in $G_{n-\ell, \ell+1}$, the domain $\mathbb{D}_{n-\ell-1, n}^{\ell}$ is disjoint with another flag domain $\Omega_{n-\ell, \ell+1} \subset$ $G_{n-\ell, \ell+1}$ and at the same time, the Shilov boundary of $\Omega_{n-\ell, \ell+1}$ is part of the boundary of $\mathbb{D}_{n-\ell-1, n}^{\ell}$. This is crucial and it makes Lemma 2.9 applicable in the present situation as in the proof of Theorem 2.11. Recall first of all the definition $\mathbb{D}_{n-\ell-1, n}^{\ell}=\left\{\left[A^{\prime}, A^{\prime \prime}\right]_{\ell} \in G_{n-\ell, \ell+1}: A^{\prime} A^{\prime H}-A^{\prime \prime} A^{\prime \prime H}>0\right\}$. On the other hand, $\Omega_{n-\ell, \ell+1}$ can be realized, using the same set of homogeneous coordinates, as the domain: $\Omega_{n-\ell, \ell+1}=\left\{\left[A^{\prime}, A^{\prime \prime}\right]_{\ell} \in G_{n-\ell, \ell+1}: A^{\prime} A^{\prime H}-A^{\prime \prime} A^{\prime \prime H}<0\right\}$. In this realization, when $\ell \geq n / 2$, the Shilov boundary is $S\left(\Omega_{n-\ell, \ell+1}\right)=\left\{\left[A^{\prime}, A^{\prime \prime}\right]_{\ell} \in G_{n-\ell, \ell+1}: A^{\prime} A^{\prime H}=\right.$ $\left.A^{\prime \prime} A^{\prime \prime H}\right\}$ and we immediately see that it is contained in the boundary of $\mathbb{D}_{n-\ell-1, n}^{\ell}$. From this point on, the proof of Theorem 2.11 can be carried over here, verbatim.

2.3. Local characterization of standard embeddings. In this section, we will give the local versions of Theorems 2.11 and 2.12 in the previous section. We will deal with the situation where a local embedding $f$ of a connected open set $U$ of $\mathbb{P}^{n}$ into $\mathbb{P}^{m}$ is given and $f$ preserves the boundary of $\mathbb{D}_{n}^{\ell}$. This is clearly relevant to the study of local proper holomorphic maps among the flag domains $\mathbb{D}_{n}^{\ell}$ as such a local proper map automatically extends across the boundary by Hartogs' extension and the extension will preserve the boundary. On the other hand, in the equidimensional case, we may also regard the theorem as a counterpart of Alexander's theorem [4] on the local characterization of the automorphisms of the unit ball in the complex Euclidean space of dimension at least two. We start with a lemma in linear algebra.

Lemma 2.13. Let $2 \leq n \leq m$ and $V, W$ be complex vector spaces of dimensions $n, m$ respectively. Suppose $V$ and $W$ are equipped with non-degenerate Hermitian forms $H_{V}(\cdot, \cdot)$ and $H_{W}(\cdot, \cdot)$ of signatures $(p, n-p)$ and $(p, m-p)$ respectively, where $1 \leq p \leq n-1$. Let $A: V \rightarrow W$ be a linear map which preserves the null vectors. Suppose furthermore that there exists $v_{+} \in V$ with $H_{V}\left(v_{+}, v_{+}\right)>0$ such that $H_{W}\left(A\left(v_{+}\right), A\left(v_{+}\right)\right)>0$. Then $A^{*} H_{W}=\lambda H_{V}$ for some $\lambda>0$.

Proof. Let $\|\cdot\|_{V},\|\cdot\|_{W}$ be the indefinite norms induced by $H_{V}$ and $H_{W}$ respectively. We fix an orthonormal basis $\left\{v_{1}, \ldots, v_{n}\right\}$ for $V$ such that $\left\|v_{i}\right\|_{V}=1$ for $1 \leq i \leq p$ and $\left\|v_{j}\right\|_{V}=-1$ for $p+1 \leq j \leq n$. Now fix $j \in\{p+1, \ldots, n\}$. The vector $v_{1}+e^{i \theta} v_{j}$ is null and hence $\left\|A\left(v_{1}+e^{i \theta} v_{j}\right)\right\|_{W}^{2}=0$ for any $\theta \in \mathbb{R}$. Then, we have

$$
H_{W}\left(A v_{1}, A v_{1}\right)+e^{i \theta} H_{W}\left(A v_{j}, A v_{1}\right)+e^{-i \theta} H_{W}\left(A v_{1}, A v_{j}\right)+H_{W}\left(A v_{j}, A v_{j}\right)=0
$$

for any $\theta \in \mathbb{R}$. From this we readily deduce that $H_{W}\left(A v_{1}, A v_{1}\right)+H_{W}\left(A v_{j}, A v_{j}\right)=0$ and $H_{W}\left(A v_{1}, A v_{j}\right)=0$. Similarly, we have $H_{W}\left(A v_{i}, A v_{i}\right)+H_{W}\left(A v_{j}, A v_{j}\right)=0$ and $H_{W}\left(A v_{i}, A v_{j}\right)=0$ for any $i \in\{1, \ldots, p\}$ and $j \in\{p+1, \ldots, n\}$. Therefore, if we let $\left\|A\left(v_{1}\right)\right\|_{W}^{2}=\lambda$ for some $\lambda \in \mathbb{R}$, we will have $A^{*} H_{W}=\lambda H_{V}$. Finally, $\lambda>0$ because $\left\|A\left(v_{+}\right)\right\|_{W}>0$ for some $v_{+} \in V$ with $\left\|v_{+}\right\|_{V}>0$.

We are now ready to give the proof for Theorem 1.1 in the case of $k=0$. 
Theorem 2.14. Let $1 \leq \ell \leq n-2$ and $U \subset \mathbb{P}^{n}$ be a connected open set such that $U \cap \partial \mathbb{D}_{n}^{\ell} \neq \emptyset$. Suppose $f: U \rightarrow \mathbb{P}^{m}$ is a holomorphic embedding in one of the following cases:

(i) $1 \leq \ell<\frac{n}{2}, f\left(U \cap \partial \mathbb{D}_{n}^{\ell}\right) \subset \partial \mathbb{D}_{m}^{\ell} ;$

(ii) $\frac{n}{2} \leq \ell \leq n-2, f\left(U \cap \partial \mathbb{D}_{n}^{\ell}\right) \subset \partial \mathbb{D}_{m}^{\ell+m-n}$,

then $f$ extends to a linear embedding of $\mathbb{P}^{n}$ into $\mathbb{P}^{m}$. If furthermore, we have in case (i) $f\left(U \cap \mathbb{D}_{n}^{\ell}\right) \subset \mathbb{D}_{m}^{\ell}$ (resp. in case (ii) $\left.f\left(U \cap \mathbb{D}_{n}^{\ell}\right) \subset \mathbb{D}_{m}^{\ell+m-n}\right)$, then the extension restricting on $\mathbb{D}_{n}^{\ell}$ is a proper holomorphic embedding into $\mathbb{D}_{m}^{\ell}$ (resp. into $\mathbb{D}_{m}^{\ell+m-n}$ ).

Proof. We will again make use of Lemma 2.10. To apply the lemma, we need to first prove that for a general line $L \subset \mathbb{P}^{n}$ intersecting $U, f(L \cap U)$ is an open subset of some line in $\mathbb{P}^{m}$.

We will deal with case $(i)$ first. Consider the $\ell$-Grassmann bundle of $U$, i.e., $G_{\ell} T U$ which is a connected open subset of $G_{\ell} T \mathbb{P}^{n}$. Recall that the holomorphic $\mathbb{P}^{\ell}$-bundle $\pi^{\text {uni }}: G_{\ell} T \mathbb{P}^{n} \rightarrow G_{\ell+1, n-\ell}$ is the universal family of $\ell$-planes in $\mathbb{P}^{n}$ and $\pi^{\mathrm{uni}}\left(G_{\ell} T U\right)$ is the set of $\ell$-planes whose intersections with $U$ are non-empty. Also recall that by Proposition 2.3 the fibers over $\overline{\mathbb{D}}_{\ell, n}^{\ell} \cong \bar{\Omega}_{\ell+1, n-\ell} \subset G_{\ell+1, n-\ell}$ in the universal family $\pi^{\text {uni }}: G_{\ell} T \mathbb{P}^{n} \rightarrow G_{\ell+1, n-\ell}$ correspond to those $\ell$-planes contained in $\overline{\mathbb{D}}_{n}^{\ell}$. Furthermore, the fibers over the Shilov boundary $S\left(\Omega_{\ell+1, n-\ell}\right)$ correspond to those lying inside $\partial \mathbb{D}_{n}^{\ell}$ by Proposition 2.5. Therefore, $U \cap \partial \mathbb{D}_{n}^{\ell} \neq \emptyset$ implies that $\pi^{\text {uni }}\left(G_{\ell} T U\right) \cap S\left(\Omega_{\ell+1, n-\ell}\right) \neq \emptyset$.

Now for each point $b \in \pi^{\text {uni }}\left(G_{\ell} T U\right) \cap S\left(\Omega_{\ell+1, n-\ell}\right)$, denote by $L_{b}$ the $\ell$-plane in $\partial \mathbb{D}_{n}^{\ell}$ corresponding to $b$. By the assumption that $f\left(U \cap \partial \mathbb{D}_{n}^{\ell}\right) \subset \partial \mathbb{D}_{m}^{\ell}$ and Lemma 2.4, we see that $f\left(U \cap L_{b}\right)$ is an open subset of some $\ell$-plane $L_{b^{\sharp}}$ lying inside $\partial \mathbb{D}_{m}^{\ell}$. Then using exactly the same argument given in the proof of Theorem 2.11 involving taking derivatives along the fibre direction and applying Lemma 2.9, we can conclude that for any $\ell$-plane $L_{z}$ corresponding to a point $z \in \pi^{\mathrm{uni}}\left(G_{\ell} T U\right), f\left(U \cap L_{z}\right)$ is an open subset of some $\ell$-plane $L_{z^{\sharp}}$ in $\mathbb{P}^{m}$. In other words, $f$ maps the intersection of any $\ell$-plane with $U$ to an open subset of an $\ell$-plane in $\mathbb{P}^{m}$. As $f$ is an embedding, $f$ also maps the intersection of any line with $U$ to an open subset of a line in $\mathbb{P}^{m}$. By Lemma 2.10, $f$ extends to a linear embedding of $\mathbb{P}^{n}$ into $\mathbb{P}^{m}$.

A linear embedding of $\mathbb{P}^{n}$ into $\mathbb{P}^{m}$ is, by definition, induced by a linear embedding $A: \mathbb{C}^{n+1} \rightarrow \mathbb{C}^{m+1}$. If we equip $\mathbb{C}^{n+1}$ with the standard non-degenerate Hermitian form $H_{\ell+1}$ of signature $(\ell+1, n-\ell)$, the points on $\mathbb{D}_{n}^{\ell}$ and on $\partial \mathbb{D}_{n}^{\ell}$ are then the lines on which the restrictions of $H_{\ell+1}$ are positive definite and zero respectively. On the other hand, by the Identity Theorem for real analytic functions, the extension of $f$ maps the whole $\partial \mathbb{D}_{n}^{\ell}$ into $\partial \mathbb{D}_{m}^{\ell}$ and therefore the corresponding linear map $A$ maps null vectors to null vectors. Now if furthermore we have $f\left(U \cap \mathbb{D}_{n}^{\ell}\right) \subset \mathbb{D}_{m}^{\ell}$, by applying Lemma 2.13 to $A$ it follows immediately that the extension of $f$ restricting on $\mathbb{D}_{n}^{\ell}$ is a proper holomorphic embedding into $\mathbb{D}_{m}^{\ell}$. This completes the proof for case (i).

For case (ii), let $\ell^{\prime}=n-\ell-1$. Then we have $1 \leq \ell^{\prime}<n / 2$. Observe that the complement of $\overline{\mathbb{D}}_{n}^{\ell}$ in $\mathbb{P}^{n}$ is just $\mathbb{D}_{n}^{\ell^{\prime}}$ by a change of coordinates. Thus, $\partial \mathbb{D}_{n}^{\ell} \cong \partial \mathbb{D}_{n}^{\ell^{\prime}}$ and similarly $\partial \mathbb{D}_{m}^{\ell+m-n} \cong \partial \mathbb{D}_{m}^{\ell^{\prime}}$ and we are back to case (i). 


\section{On Grassmannians of higher rank}

3.1. Confined sub-Grassmannians in $\overline{\mathbb{D}}_{k, n}^{\ell}$. We are going to generalize the results obtained in the previous sections to the flag domains $\mathbb{D}_{k, n}^{\ell} \subset G_{k+1, n-k}, k \geq 1$ and $\ell \in\{k+1, \ldots, n-k-2\}$. This time we will look at the sub-Grassmannians in $G_{k+1, n-k}$ corresponding to linear subspaces in $\mathbb{C}^{n+1}$. More explicitly, for a linear subspace $E \subset \mathbb{C}^{n+1}$ with $\operatorname{dim}(E)=e+1 \geq k+1$, we consider the Grassmannian $G_{k+1, e-k}^{E}:=\{(k+1)$-planes contained in $E\}$, which is a totally geodesic submanifold of $G_{k+1, n-k}$ with respect to any choice of canonical Kähler-Einstein metric. We will call it an $(e+1)$-confined sub-Grassmannian or simply a confined sub-Grassmannian in this paper. It is clearly a generalization of the projective linear subspaces in $\mathbb{P}^{n}$.

Similar to the case $\overline{\mathbb{D}}_{n}^{\ell} \subset \mathbb{P}^{n}$, we will first determine the set of confined subGrassmannians of $G_{k+1, n-k}$ which are contained in $\overline{\mathbb{D}}_{k, n}^{\ell}$. The following proposition is a generalization of Proposition 2.6.

Proposition 3.1. Let $k \leq e \leq \ell$. The set of $(e+1)$-confined sub-Grassmannians contained in $\mathbb{D}_{k, n}^{\ell}\left(\right.$ resp. $\left.\overline{\mathbb{D}}_{k, n}^{\ell}\right)$ is parametrized by $\mathbb{D}_{e, n}^{\ell}\left(\right.$ resp. $\left.\overline{\mathbb{D}}_{e, n}^{\ell}\right)$. Furthermore, among them the $(\ell+1)$-confined sub-Grassmannians are maximal compact complex-analytic subvarieties in $\mathbb{D}_{k, n}^{\ell}$.

Proof. The proof is parallel to those of Propositions 2.2 and 2.6 and we will just simply write down the explicit parametrization. Take a point $E \in \mathbb{D}_{e, n}^{\ell}\left(\right.$ resp. $\overline{\mathbb{D}}_{e, n}^{\ell}$ ) and choose a matrix representative in homogeneous coordinates $\left[E^{\prime}, E^{\prime \prime}\right]_{\ell}$ for $E$. The corresponding $(e+1)$-confined sub-Grassmannian is $G_{k+1, e-k}^{E}=\left\{\left[Z E^{\prime}, Z E^{\prime \prime}\right]_{\ell} \in\right.$ $\left.G_{k+1, n-k}:[Z] \in G_{k+1, e-k}\right\}$ and it is contained in $\mathbb{D}_{k, n}^{\ell}\left(\right.$ resp. $\left.\overline{\mathbb{D}}_{k, n}^{\ell}\right)$ because $E \in \mathbb{D}_{e, n}^{\ell}$ (resp. $\overline{\mathbb{D}}_{e, n}^{\ell}$ ).

Recall from the introduction section that we will always consider the cases where $k+1 \leq \ell \leq n-k-2$. We now analyze the boundary $\partial \mathbb{D}_{k, n}^{\ell}$ as in Lemma 2.4 and Proposition 2.5. The difference here is that $\partial \mathbb{D}_{k, n}^{\ell}$ is no longer homogeneous for $k \geq 1$ and it actually consists of $k+1$ strata. They can be described as follows. For each $r \in\{1, \ldots, k+1\}$, the $r$ th stratum of $\partial \mathbb{D}_{k, n}^{\ell}$, denoted by $\partial_{r} \mathbb{D}_{k, n}^{\ell}$, is defined to be the set of $\left[Z^{\prime}, Z^{\prime \prime}\right]_{\ell} \in G_{k+1, n-k}$ such that $Z^{\prime} Z^{\prime H}-Z^{\prime \prime} Z^{\prime \prime H} \geq 0$ with the null space being of dimension $r$. For our purpose, the most important stratum is $\Sigma\left(\mathbb{D}_{k, n}^{\ell}\right):=\partial_{k+1} \mathbb{D}_{k, n}^{\ell}$ which can be defined equivalently as the set $\left\{\left[Z^{\prime}, Z^{\prime \prime}\right]_{\ell} \in\right.$ $\left.G_{k+1, n-k}: Z^{\prime} Z^{\prime H}=Z^{\prime \prime} Z^{\prime \prime H}\right\}$.

Proposition 3.2. Let $k+1 \leq \ell \leq n-k-2$. The maximal compact complexanalytic subvarieties in $\Sigma\left(\mathbb{D}_{k, n}^{\ell}\right)$ are $(\ell+1)$-confined subgrassmannians when $\ell<$ $n / 2$ and $(n-\ell)$-confined sub-Grassmannians when $\ell \geq n / 2$. Moreover, any germ of a complex submanifold lying inside $\Sigma\left(\mathbb{D}_{k, n}^{\ell}\right)$ must be contained in one of these sub-Grassmannians.

Proof. We first note that $\Sigma\left(\mathbb{D}_{k, n}^{\ell}\right)$ and $\Sigma\left(\mathbb{D}_{k, n}^{n-\ell-1}\right)$ are equivalent under an automorphism of $G_{k+1, n-k}$. To see this, consider the domain defined by $D=\left\{\left[Z^{\prime}, Z^{\prime \prime}\right]_{\ell} \in\right.$ $\left.G_{k+1, n-k}: Z^{\prime} Z^{\prime H}-Z^{\prime \prime} Z^{\prime \prime H}<0\right\}$. It is clearly biholomorphic to $\mathbb{D}_{k, n}^{n-\ell-1}$ under the automorphism of $G_{k+1, n-k}$ given by $\left[Z^{\prime}, Z^{\prime \prime}\right]_{\ell} \mapsto\left[Z^{\prime \prime}, Z^{\prime}\right]_{n-\ell-1}$. Now it suffices to 
know that both $\Sigma(D)$ and $\Sigma\left(\mathbb{D}_{k, n}^{\ell}\right)$ are defined by the equation $Z^{\prime} Z^{\prime H}=Z^{\prime \prime} Z^{\prime \prime H}$. Thus, we just need to prove the proposition for $\ell<n / 2$.

Suppose we are given a germ of a holomorphic curve $g: \Delta \rightarrow \Sigma\left(\mathbb{D}_{k, n}^{\ell}\right)$, where $\Delta$ is the unit disk. Without loss of generality, we may assume that $g(\Delta)$ lies in in the Euclidean cell $U:=\left\{\left[Z^{\prime}, Z^{\prime \prime}\right]_{k} \in G_{k+1, n-k}: \operatorname{det}\left(Z^{\prime}\right) \neq 0\right\} \cong \mathbb{C}^{(k+1)(n-k)}$ and in the standard inhomogeneous coordinates of $U$, we can write $g(z)=\left(G^{\prime}(z), G^{\prime \prime}(z)\right)$, $G^{\prime}(z) \in M(k+1, \ell-k ; \mathbb{C}), G^{\prime \prime}(z) \in M(k+1, n-\ell ; \mathbb{C})$ and so that for every $z$, $\left[\left(I_{k+1}, G^{\prime}(z)\right), G^{\prime \prime}(z)\right]_{\ell}$ is a matrix representative in homogeneous coordinates for $g(z)$, where $I \in M(k+1 ; \mathbb{C})$ is the identity matrix. Then $g(\Delta) \subset \Sigma\left(\mathbb{D}_{k, n}^{\ell}\right)$ implies that $\left(I, G^{\prime}\right)\left(I, G^{\prime}\right)^{H}=G^{\prime \prime} G^{\prime H}$ and hence there exists a matrix $A \in M(\ell+1, n-\ell ; \mathbb{C})$, $A A^{\mathrm{H}}=I$, such that $G^{\prime \prime}=\left(I, G^{\prime}\right) A$. Therefore, $g(\Delta)$ is contained in the $(\ell+1)$ confined sub-Grassmannian $G_{k+1, \ell-k}^{\left[I_{\ell+1}, A\right]_{\ell}}=\left\{[Z, Z A]_{\ell} \in G_{k+1, n-k}:[Z] \in G_{k+1, \ell-k}\right\}$. Note that $G_{k+1, \ell-k}^{\left[I_{\ell+1}, A\right]_{\ell}} \subset \Sigma\left(\mathbb{D}_{k, n}^{\ell}\right)$. Replacing a germ of a curve in the above argument by a germ of a holomorphic map from a higher-dimensional unit ball into $\Sigma\left(\mathbb{D}_{k, n}^{\ell}\right)$, we conclude similarly that any germ of a complex submanifold inside $\Sigma\left(\mathbb{D}_{k, n}^{\ell}\right)$ must be contained in some $(\ell+1)$-confined sub-Grassmannian in $\Sigma\left(\mathbb{D}_{k, n}^{\ell}\right)$. In particular, for $A \in M(\ell+1, n-\ell ; \mathbb{C})$ with $A A^{\mathrm{H}}=I$, the $(\ell+1)$-confined sub-Grassmannians $G_{k+1, \ell-k}^{[I, A]}$ are maximal compact complex-analytic subvarieties of $\Sigma\left(\mathbb{D}_{k, n}^{\ell}\right)$.

3.1.1. Double fibrations. Fix one $G_{k+1, n-k}$ and take a point $p \in G_{k+1, n-k}$. Let $e \in\{k, \ldots, n\}$. An $(e+1)$-confined sub-Grassmannian passing through $p$ will give one $(k+1)(e-k)$-dimensional tangent subspace in $T_{p} G_{k+1, n-k}$, or equivalently, a point in the Grassmannian of $(k+1)(e-k)$-planes in $T_{p} G_{k+1, n-k}$, i.e., $G_{(k+1)(e-k)} T_{p} G_{k+1, n-k}$ (see the definitions before Proposition 2.8). The collection of all such $(k+1)(e-$ $k$ )-dimensional tangent subspaces at $p$ gives a complex-analytic subvariety $\mathfrak{F}_{p}^{e}$ of $G_{(k+1)(e-k)} T_{p} G_{k+1, n-k}$. This can be seen by noting that $\mathfrak{F}_{p}^{e}$ is an orbit of the isotropy action of some parabolic subgroup of $S L(n+1, \mathbb{C})$ on $G_{(k+1)(e-k)} T_{p} G_{k+1, n-k}$. Within a Euclidean cell of $G_{k+1, n-k}$, any Euclidean translation extends to a biholomorphism of $G_{k+1, n-k}$ and hence $\mathfrak{F}_{p}^{e}$ at any point is simply the Euclidean translation of $\mathfrak{F}_{0}^{e}$ at the origin. Let us write $\mathfrak{F}^{e}:=\bigcup_{p \in G_{k+1, n-k}} \mathfrak{F}_{p}^{e}$. Since a confined sub-Grassmannian is uniquely determined by its tangent space at one point, we have therefore a holomorphic $G_{k+1, e-k}$-fibration of $\mathfrak{F}^{e}$. Moreover, as the set of $(e+1)$-confined sub-Grassmannians in $G_{k+1, n-k}$ is in one-to-one correspondence to the set of $(e+1)$-planes in $\mathbb{C}^{n+1}$, we have the universal family $\mathfrak{F}^{e} \rightarrow G_{e+1, n-e}$ as a holomorphic $G_{k+1, e-k}$-bundle.

When $p \in \mathbb{D}_{k, n}^{\ell}$, for each $e \in\{k, \ldots, \ell\}$, if we restrict to those $(e+1)$-confined sub-Grassmannians contained in $\mathbb{D}_{k, n}^{\ell}$, then their tangent spaces constitute an open subset $\mathfrak{D}_{p}^{e} \subset \mathfrak{F}_{p}^{e}$ and we let $\mathfrak{D}^{e}=\bigcup_{p \in \mathbb{D}_{k, n}^{\ell}} \mathfrak{D}_{p}^{e}$. The foliation of $\mathfrak{F}^{e}$ restricts to a foliation of $\mathfrak{D}^{e}$ and we have the universal family $\mathfrak{D}^{e} \rightarrow \mathbb{D}_{e, n}^{\ell}$ as a holomorphic $G_{k+1, e-k}$-bundle.

We now restrict to the case $e=\ell$. As in the case of $\mathbb{D}_{n}^{\ell}$, we insert one more double fibration between the double fibrations of $\mathfrak{D}^{\ell}$ and $\mathfrak{F}^{\ell}$. Let $U^{\ell}=\left\{\left[Z^{\prime}, Z^{\prime \prime}\right]_{\ell} \in G_{k+1, n-k}\right.$ : $\left.\operatorname{det}\left(Z^{\prime}\right) \neq 0\right\}$. Then the $(\ell+1)$-confined sub-Grassmannian $\left\{\left[Z A^{\prime}, Z A^{\prime \prime}\right]_{\ell} \in G_{k+1, n-k}\right.$ : $\left.[Z] \in G_{k+1, \ell-k}\right\}$ is contained in $U^{\ell}$ for any $\left[A^{\prime}, A^{\prime \prime}\right]_{\ell} \in G_{\ell+1, n-\ell}$ with $\operatorname{det}\left(A^{\prime}\right) \neq 0$. Note that the set $\left\{\left[A^{\prime}, A^{\prime \prime}\right]_{\ell} \in G_{\ell+1, n-\ell}: \operatorname{det}\left(A^{\prime}\right) \neq 0\right\} \cong \mathbb{C}^{(\ell+1)(n-\ell)}$. Then similar to $\mathbb{D}_{k, n}^{\ell}$, we can find an open subset $\mathfrak{W}^{\ell} \subset \mathfrak{F}^{\ell}$ with $\pi_{\ell}\left(\mathfrak{W}^{\ell}\right)=U^{\ell}$, where $\pi_{\ell}$ is the canonical 
projection, such that the projection onto the cycle space, $\mathfrak{W}^{\ell} \rightarrow \mathbb{C}^{(\ell+1)(n-\ell)}$, is a holomorphic $G_{k+1, \ell-k}$-bundle. In summary, we have the following inclusion relations:

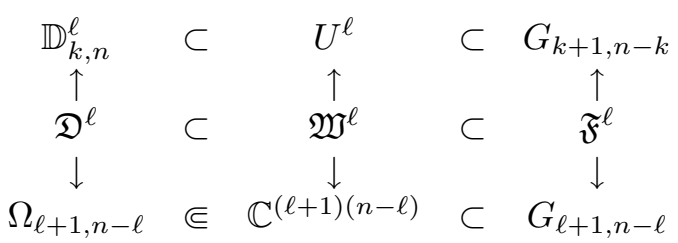

3.2. Local characterization of standard embeddings. We will make use of the following Cartan-Fubini-type extension theorem of Mok [3] on holomorphic maps between Grassmannians. To state and apply the theorem, we need to recall a few basic facts about Grassmannians. Since $G_{m, n}$ is biholomorphic to $G_{n, m}$ we will assume that $m \leq n$. Each $G_{m, n}$ can be embedded into the projective space $\mathbb{P} \Lambda^{m}\left(\mathbb{C}^{m+n}\right)$ by the Plücker embedding. The image of $G_{m, n}$ in $\mathbb{P} \Lambda^{m}\left(\mathbb{C}^{m+n}\right)$ contains certain $m$-dimensional projective linear subspaces in $\mathbb{P} \Lambda^{m}\left(\mathbb{C}^{m+n}\right)$ corresponding to the $(m+1)$-confined sub-Grassmannians $G_{m, m+1} \cong \mathbb{P}^{m}$. In addition, there is a notion of minimal rational curves for $G_{m, n}$ (see [10]). In the present context, they are the lines in $\mathbb{P} \Lambda^{m}\left(\mathbb{C}^{m+n}\right)$ that lie inside the image of $G_{m, n}$ under the Plücker embedding. In fact, they are just the lines in those $m$-dimensional projective linear subspaces we have just described. At each point $p \in G_{m, n}$, a tangent vector is called a minimal characteristic vector or minimal rational tangent if it is tangent to some minimal rational curve in $G_{m, n}$. The set of projectivized minimal rational tangents at $p$ is called the variety of minimal rational tangents (VMRT) at $p$, denoted by $\mathcal{C}_{p}$. At each point $p \in G_{m, n}$, the VMRT $\mathcal{C}_{p} \subset \mathbb{P} T_{p} G_{m, n} \cong \mathbb{P}^{m n-1}$ can be identified as the image of the Segre embedding $\mathbb{P}^{m-1} \times \mathbb{P}^{n-1} \rightarrow \mathbb{P}^{m n-1}$.

Theorem 3.3 (Mok). Let $G$ and $G^{\prime}$ be Grassmannians of rank at least 2. Let $h$ : $U \subset G \rightarrow G^{\prime}$ be a local holomorphic map defined on some connected open set $U$ and let $d h$ be its differential. If dh maps minimal characteristic vectors to minimal characteristic vectors and $h(U)$ is not contained in any projective linear subspace in $G^{\prime}$, then $h$ extends to a standard embedding of $G$ into $G^{\prime}$.

In the present context, a standard embedding of $G_{k+1, n-k}$ into $G_{k+1, m-k}$ means an embedding of $G_{k+1, n-k}$ into $G_{k+1, m-k}$ induced by a linear embedding $A: \mathbb{C}^{n+1} \rightarrow$ $\mathbb{C}^{m+1}$. We also remark that a standard embedding between Grassmannians is totally geodesic with respect to any choice of Kähler metric that define the relevant Grassmannians as Hermitian symmetric spaces. The choice of the Kähler metric on $G_{k+1, n-k}$ corresponds to a choice of Euclidean metric on $\mathbb{C}^{n+1}$.

The proof of the following proposition is along a similar approach as in the case of $\mathbb{D}_{n}^{\ell}$.

Proposition 3.4. Let $k+1 \leq \ell<n / 2$. Let $U \subset G_{k+1, n-k}$ be a connected open set and $U \cap \Sigma\left(\mathbb{D}_{k, n}^{\ell}\right) \neq \emptyset$. Let $f: U \rightarrow G_{k+1, m-k}$ be a holomorphic embedding and $f\left(U \cap \Sigma\left(\mathbb{D}_{k, n}^{\ell}\right)\right) \subset \Sigma\left(\mathbb{D}_{k, m}^{\ell}\right)$. Then the differential df maps minimal characteristic vectors to minimal characteristic vectors.

Proof. We recall the notations in Section 3.1.1. The holomorphic $G_{k+1, \ell-k}$-bundle $\pi^{u n i}: \mathfrak{F}^{\ell} \rightarrow G_{\ell+1, n-\ell}$ is the universal family of $(\ell+1)$-confined sub-Grassmannians 
in $G_{k+1, n-k}$. Let $\mathfrak{F}_{U}^{\ell}:=\bigcup_{p \in U} \mathfrak{F}_{p}^{\ell}$. It is an open subset of $\mathfrak{F}^{\ell}$ and $\pi^{\text {uni }}\left(\mathfrak{F}_{U}^{\ell}\right)$ are precisely those sub-Grassmannians intersecting $U$. Moreover, with respect to the standard homogeneous coordinates in $G_{\ell+1, n-\ell}$, the fibers over $\overline{\mathbb{D}}_{\ell, n}^{\ell} \cong \bar{\Omega}_{\ell+1, n-\ell} \subset G_{\ell+1, n-\ell}$ are those contained in $\overline{\mathbb{D}}_{k, n}^{\ell}$ and the fibers over the Shilov boundary $S\left(\Omega_{\ell+1, n-\ell}\right)$ are those contained in $\Sigma\left(\mathbb{D}_{k, n}^{\ell}\right)$. Since $U \cap \Sigma\left(\mathbb{D}_{k, n}^{\ell}\right) \neq \emptyset$, we have $\pi^{u n i}\left(\mathfrak{F}_{U}^{\ell}\right) \cap S\left(\Omega_{\ell+1, n-\ell}\right) \neq \emptyset$.

Now take a point $b \in U \cap \Sigma\left(\mathbb{D}_{k, n}^{\ell}\right)$ and $v_{b} \in \mathfrak{F}_{b}^{\ell}$ such that $\pi^{u n i}\left(v_{b}\right) \in S\left(\Omega_{\ell+1, n-\ell}\right)$.

In the target space, by assumption, we have $f(b) \in \Sigma\left(\mathbb{D}_{k, m}^{\ell}\right)$ and by Proposition 3.2, $d f\left(v_{b}\right) \in \mathfrak{F}_{f(b)}^{\ell}$. We now choose a connected open neighborhood $Y \subset G_{k+1, m-k}$ of $f(b)$ and a connected open neighborhood $\Upsilon \subset G_{(k+1)(\ell-k)} T_{f(b)} G_{k+1, m-k}$ of $d f\left(v_{b}\right)$. We may choose $Y$ and $\Upsilon$ small enough so that $Y$ (resp. $\Upsilon$ ) lies in some Euclidean cell of $G_{k+1, m-k}$ (resp. $G_{(k+1)(\ell-k)} T_{f(b)} G_{k+1, m-k}$ ) and $\Upsilon \cap \mathfrak{F}_{f(b)}^{\ell}$ is precisely the zero set of a family of holomorphic functions $\left\{h_{i}\right\}_{i \in I}$ on $\Upsilon$. Recall that each fibre in $\mathfrak{F}_{Y}^{\ell}:=$ $\bigcup_{y \in Y} \mathfrak{F}_{y}^{\ell} \rightarrow Y$ is just the Euclidean parallel transport of one another. Thus, $\mathfrak{F}_{Y}^{\ell} \cap(Y \times$ $\Upsilon)$ is also precisely the zero set of $\left\{h_{i}\right\}_{i \in I}$ when these functions are trivially extended from $\Upsilon$ to $Y \times \Upsilon$.

Back to the domain, we choose small enough connected open sets $\mathcal{W} \subset \pi^{u n i}\left(\mathfrak{F}_{U}^{\ell}\right)$ and $\mathbf{W} \subset G_{k+1, \ell-k}$ with the following properties: (1) $v_{b} \in \mathcal{W} \times \mathbf{W} ;(\mathbf{2}) d f(\mathcal{W} \times \mathbf{W}) \subset$ $Y \times \Upsilon$ and $(\mathbf{3}) \mathcal{W}$ (resp. W) lies in some Euclidean cell of $G_{\ell+1, n-\ell}\left(\right.$ resp. $\left.G_{k+1, \ell-k}\right)$. (In particular, $\mathcal{W} \cap S\left(\Omega_{\ell+1, n-\ell}\right) \neq \emptyset$. ) We pull back the functions $\left\{h_{i}\right\}_{i \in I}$ from $Y \times \Upsilon$ to $\mathcal{W} \times \mathbf{W}$ by $d f$ and denote the pulled-back functions by $\left\{h_{i}^{*}\right\}_{i \in I}$.

Take a section $\mathcal{W}_{\mathbf{w}}:=\mathcal{W} \times\{\mathbf{w}\} \subset \mathcal{W} \times \mathbf{W}$ and $\mathcal{W}_{\mathbf{w}}$ can be identified as a connected open set in $\mathbb{C}^{(\ell+1)(n-\ell)}$ whose intersection with $S\left(\Omega_{\ell+1, n-\ell}\right)$ is non-empty. Now we claim that each $h_{i}^{*}$ is zero on $\mathcal{W}_{\mathbf{w}} \cap S\left(\Omega_{\ell+1, n-\ell}\right)$ and hence identically zero on $\mathcal{W}_{\mathbf{w}}$ by Lemma 2.9. To see this, note that by construction, each point $w \in \mathcal{W}_{\mathbf{w}} \cap S\left(\Omega_{\ell+1, n-\ell}\right)$ is the tangent plane at a point $b_{w} \in \Sigma\left(\mathbb{D}_{k, n}^{\ell}\right)$ of some $(\ell+1)$-confined sub-Grassmannian in $G_{k+1, n-k}$ that completely lies inside $\Sigma\left(\mathbb{D}_{k, n}^{\ell}\right)$. By Proposition $3.2, d f(w) \in \mathfrak{F}_{f\left(b_{w}\right)}^{\ell}$ and hence we have $h_{i}^{*}(w)=0$ for all $i \in I$ and thereby we have the claim. Since $\mathbf{w} \in \mathbf{W}$ is arbitrary, we then have $h_{i}^{*} \equiv 0$ on $\mathcal{W} \times \mathbf{W}$ for every $i \in I$. It is equivalent to saying that for every $w \in \mathcal{W} \times \mathbf{W}, d f(w) \in \mathfrak{F}^{\ell}$, where $\mathfrak{F}^{\ell} \rightarrow G_{k+1, m-k}$ is the corresponding bundle in the target. As $\mathcal{W} \times \mathbf{W} \subset \mathfrak{F}_{U}^{\ell}$ is open and $\mathfrak{F}_{U}^{\ell}$ is connected, by combining with the standard connectedness argument we conclude that $d f\left(\mathfrak{F}_{U}^{\ell}\right) \subset \mathfrak{F}^{\ell}$.

Note that any non-empty intersection of two confined-sub-Grassmannians is again a confined sub-Grassmannian and any $(k+2)$-confined sub-Grassmannian is the intersection of a set of $(\ell+1)$-confined sub-Grassmannians (recall that $\ell \geq k+1$ ). Since $f$ is an embedding, we therefore see that $d f\left(\mathfrak{F}_{U}^{k+1}\right) \subset \mathfrak{F}^{k+1}$, where $\mathfrak{F}^{k+1} \rightarrow G_{k+1, m-k}$ is the corresponding bundle in the target. Finally, as have been mentioned in the beginning of this section, the minimal characteristic vectors on $G_{k+1, n-k}$ or $G_{k+1, m-k}$ are just those tangent vectors tangent to any of the $(k+2)$-confined sub-Grassmannians. Hence, $d f$ maps minimal characteristic vectors to minimal characteristic vectors. The proof is complete.

Corollary 3.5. Let $\frac{n}{2} \leq \ell \leq n-k-2$ and $U \subset G_{k+1, n-k}$ be a connected open set, $U \cap \Sigma\left(\mathbb{D}_{k, n}^{\ell}\right) \neq \emptyset$. Let $f: U \rightarrow G_{k+1, m-k}$ be a holomorphic embedding and 
$f\left(U \cap \Sigma\left(\mathbb{D}_{k, n}^{\ell}\right)\right) \subset \Sigma\left(\mathbb{D}_{k, m}^{\ell+m-n}\right)$. Then the differential df maps minimal characteristic vectors to minimal characteristic vectors.

Proof. Recall that $\Sigma\left(\mathbb{D}_{k, n}^{\ell}\right)$ and $\Sigma\left(\mathbb{D}_{k, n}^{n-\ell-1}\right)$ are equivalent under an automorphism of $G_{k+1, n-k}$. Similarly, $\Sigma\left(\mathbb{D}_{k, m}^{\ell+m-n}\right)$ and $\Sigma\left(\mathbb{D}_{k, m}^{n-\ell-1}\right)$ are equivalent. Let $\ell^{\prime}=n-\ell-1$, then we have $k+1 \leq \ell^{\prime}<n / 2$ and $f\left(U \cap \Sigma\left(\mathbb{D}_{k, n}^{\ell^{\prime}}\right)\right) \subset \Sigma\left(\mathbb{D}_{k, m}^{\ell^{\prime}}\right)$. Now the result follows from Proposition 3.4.

We are finally in the position the prove Theorem 1.1 for $k \geq 1$.

Proof. We first prove that a local holomorphic embedding $f: U \subset G_{k+1, n-k} \rightarrow$ $G_{k+1, m-k}$ satisfying the hypotheses extends to a standard embedding of $G_{k+1, n-k}$ into $G_{k+1, m-k}$. By Proposition 3.4, Corollary 3.5 and Theorem 3.3, we just need to check that $f(U)$ is not contained in any projective linear subspace in $G_{k+1, m-k}$. There are two kinds of projective linear subspaces in $G_{k+1, m-k}$. The first kind is the $(k+2)$-confined sub-Grassmannians. They are biholomorphic to $\mathbb{P}^{k+1}$. Since $f$ is a local embedding, by counting the dimensions we see that this is impossible. The second kind can be described as follows. Let $V \subset \mathbb{C}^{m+1}$ be a $k$-dimensional linear subspace and $W$ be a complementary linear subspace of $V$ in $\mathbb{C}^{m+1}$. Then the set $\left\{E \in G_{k+1, m-k}: E=V \oplus w, w \in \mathbb{P}(W)\right\}$ is an $(m-k)$-dimensional projective linear subspace in $G_{k+1, m-k}$ and we denote it by $\mathbb{P}_{V, W}^{m-k}$.

Suppose $f(U) \subset \mathbb{P}_{V, W}^{m-k}$ for some $V, W \subset \mathbb{C}^{m+1}$. We will first consider case $(i)$ in the statement of Theorem 1.1. Recall that the maximal compact complex-analytic subvarieties in $\Sigma\left(\mathbb{D}_{k, n}^{\ell}\right)$ and $\Sigma\left(\mathbb{D}_{k, m}^{\ell}\right)$ are $(\ell+1)$-confined sub-Grassmannians and are of dimension $(k+1)(\ell-k)$ (Proposition 3.2). Since $f$ is an embedding and $f(U \cap$ $\left.\Sigma\left(\mathbb{D}_{k, n}^{\ell}\right)\right) \subset \Sigma\left(\mathbb{D}_{k, m}^{\ell}\right) \cap \mathbb{P}_{V, W}^{m-k}$ by the hypotheses, we deduce that some $(\ell+1)$-confined sub-Grassmannian in $\Sigma\left(\mathbb{D}_{k, m}^{\ell}\right)$ is contained in $\mathbb{P}_{V, W}^{m-k}$. But this is impossible because all $(k+1)$-planes parametrized by $\mathbb{P}_{V, W}^{m-k}$ contain $V$ while obviously for a non-trivial confined-sub-Grassmannian, some $(k+1)$-planes parametrized by it do not contain $V$.

If our map $f$ belongs to case (ii), we let $\ell^{\prime}=n-\ell-1$. Then we have $k+1 \leq$ $\ell^{\prime}<\frac{n}{2}$. As explained in Proposition 3.2, $\Sigma\left(\mathbb{D}_{k, n}^{\ell}\right)$ and $\Sigma\left(\mathbb{D}_{k, n}^{\ell^{\prime}}\right)$ are equivalent under an automorphism of $G_{k+1, n-k}$ and similarly for $\Sigma\left(\mathbb{D}_{k, m}^{\ell+m-n}\right)$ and $\Sigma\left(\mathbb{D}_{k, m}^{\ell^{\prime}}\right)$. We are then back to case (i) and hence case (ii) is also settled.

By the Identity Theorem for real analytic functions, the extension of $f$ maps the whole $\Sigma\left(\mathbb{D}_{k, n}^{\ell}\right)$ into $\Sigma\left(\mathbb{D}_{k, m}^{\ell}\right)$. Note that $\Sigma\left(\mathbb{D}_{k, n}^{\ell}\right)$ can be interpreted as the set of $(k+1)$-dimensional null-planes in $\mathbb{C}^{n+1}$ when the latter is equipped with the standard non-degenerate Hermitian form with signature $(\ell+1, n-\ell)$. On the other hand, the extension of $f$ is induced by some linear map $A: \mathbb{C}^{n+1} \rightarrow \mathbb{C}^{m+1}$. With the above interpretation, we see that $A$ must map null vectors in $\mathbb{C}^{n+1}$ to null vectors in $\mathbb{C}^{m+1}$. If we have furthermore $f\left(U \cap \mathbb{D}_{k, n}^{\ell}\right) \subset \mathbb{D}_{k, m}^{\ell}$ in case (i) (resp. $f\left(U \cap \mathbb{D}_{k, n}^{\ell}\right) \subset \mathbb{D}_{k, m}^{\ell+m-n}$ in case (ii)), it follows readily from Lemma 2.13 that the extension of $f$ restricts to a proper holomorphic embedding of $\mathbb{D}_{k, n}^{\ell}$ into $\mathbb{D}_{k, m}^{\ell}$ (resp. into $\mathbb{D}_{k, m}^{\ell+m-n}$ ). 


\section{Acknowledgements}

The author would like to express his gratitude to Professor Ngaiming Mok for a lot of invaluable advice and inspirations especially for his explanation on the concept of double fibration. In fact, it is also Professor Mok who first suggested giving an alternative proof for the theorem of Baouendi-Huang by looking at the subvarieties in the boundary of $\mathbb{D}_{n}^{\ell}$. The author would also like to thank Professor Xiaojun Huang for many fruitful discussions and his detailed explanation on the work with Professor M. S. Baouendi. Finally, he would like to thank the referees for reading the paper carefully and making very useful suggestions for improving the paper.

\section{References}

[1] G. Fels, A. Huckleberry and J.A. Wolf, Cycle spaces of flag domains - a complex geometric viewpoint. Birkhäuser, 2006.

[2] M.S. Baouendi and X. Huang, Super-rigidity for holomorphic mappings between hyperquadrics with positive signature, J. Diff. Geom. 69 (2005), 379-398.

[3] N. Mok, Characterization of standard embeddings between complex Grassmannians by means of varieties of minimal rational tangents, Sci. China Ser. A 4 (2008), 660-684.

[4] H. Alexander, Holomorphic mappings from the ball and polydisc, Math. Ann. 209 (1974), 249256.

[5] G.M. Henkin and A.E. Tumanov, Local characterization of analytic automorphisms of classical domains (Engl. transl.). Soviet Math. Dokl. 26 (1982), 702-705.

[6] S.-C. Ng, On holomorphic isometric embeddings of the unit disk into polydisks, Proc. Amer. Math. Soc. 138 (2010), 2907-2922.

[7] J.A. Wolf, Fine structures of Hermitian symmetric spaces, in 'Geometry of symmetric spaces' (W.M. Boothby, ed.), Marcel-Dekker, New York, 1972.

[8] N. Mok, G-structures on irreducible Hermitian symmetric spaces of rank $\geq 2$ and deformation rigidity, Contemp. Math. 222 (1999), 81-107.

[9] S.-C. Ng, Proper holomorphic mappings on flag domains of $S U(p, q)$-type on projective spaces, Preprint: http://hkumath.hku.hk/ imr/IMRPreprintSeries/2011/IMR2011-12.pdf.

[10] J.-M. Hwang and N. Mok, Varieties of minimal rational tangents on uniruled projective manifolds, in 'Several complex variables', (M. Schneider and Y.-T. Siu, eds.), vol 37, pp 351-389, Cambridge University Press, 1999.

Department of Mathematics, The University of Hong Kong, Pokfulam Road, Hong Kong

E-mail address: math.scng@gmail.com 